\title{
Cost Efficiencies and Upward Pricing Pressure
}

\author{
JÉSSica DUtra TARUn SABARWAL \\ The University of Kansas
}

First draft: December 3, 2016

This version: December 18, 2018

\begin{abstract}
We investigate the accuracy of UPP as a tool in antitrust analysis when there are cost efficiencies from a horizontal merger. We include model-based, merger-specific cost efficiencies in a tractable manner and extend the standard UPP formulation to account for these efficiencies. The efficacy of the new UPP formulations is analyzed using Monte Carlo simulation of 40,000 mergers (8 scenarios, 5,000 mergers in each scenario). We find that the new UPP formulations yield substantial gains in prediction of post-merger prices, as compared to existing practice, and there are substantial gains in merger screening accuracy as well. Moreover, the new UPP formulations outperform the standard UPP formulation at higher thresholds for all the standard cases in the paper. The results support the inclusion of model-based cost efficiencies in the standard UPP formulation for more accurate antitrust decision-making.
\end{abstract}

JEL: K21, L11, L41, L13

Keywords: upward pricing pressure, merger efficiency, monte carlo, UPP, mergers, antitrust, unilateral effects, cost efficiencies

\section{Introduction}

A central tenet in antitrust policy is that antitrust agencies want to block mergers that are anticompetitive without interfering with ones that are procompetitive. Antitrust agencies spend considerable time, effort, and resources to determine the impact a merger may have on the post-merger competitive landscape.

One important measure of anticompetitive behavior is an increase in post-merger prices, because of increased likelihood of harm to consumers from market power. Full-merger simulations to determine anticompetitive effects and post-merger prices are time consuming and expensive to implement. Both antitrust agencies and merging parties prefer to avoid this if it is not needed. This motivates a need for alternative merger screening tools that are less expensive, quick, reliable, and theoretically grounded. 
Upward pricing pressure (UPP), proposed by Farrell \& Shapiro (2010), is being used increasingly in this regard as a pre-merger screening tool to estimate unilateral anticompetitive effects in horizontal mergers. UPP is now included in the U.S. Department of Justice and the Federal Trade Commission Horizontal Merger Guidelines (2010) and used increasingly worldwide. $^{1}$

UPP measures the first impulse for a merged firm to raise prices. It is derived by comparing the first-order condition of the merged firm with that of the pre-merger firm. The standard formulation is as follows. Suppose firms $i$ and $j$ merge. Upward pricing pressure (for the good produced by firm $i$ ) is given by: $U P P_{i}=D_{i j}\left(P_{j}-M C_{j}\right)$. Here $D_{i j}$ is the diversion matrix, which measures proportion of sales lost by firm $i$ that are recaptured by firm $j$, and $\left(P_{j}-M C_{j}\right)$ is the margin for firm $j$. Both are computed at pre-merger values.

The UPP computation has several benefits. It uses information about merging firms only, not other firms in the industry, and therefore, market shares of other firms are not needed. This simplifies its computation. Moreover, it is relatively quick and easy to implement, requires less information than some other measures, and is theoretically grounded.

The UPP computation has some limitations as well. It is only a first impulse to raise prices, and says nothing about final post-merger prices or accuracy of prediction using UPP. Moreover, it does not include cost efficiencies from a merger. When the first impulse to raise prices is high, merging parties argue with antitrust agencies that the UPP calculation should be lowered, because there are cost efficiencies from the merger, but antitrust agencies argue for more realistic numbers and require additional justification. Current practice is to arrive at an ad hoc adjustment to the UPP calculation to account for cost efficiencies. Its relation to and derivation from fundamental principles is typically left unexplored.

We investigate the accuracy of UPP as a tool in antitrust analysis by extending the standard UPP formulation to include model-based, merger-specific cost efficiencies. Using the theoretical framework in Jaffe \& Weyl (2013), we include cost efficiencies in a tractable manner and derive the related UPP formulations.

The efficacy of the new UPP formulations is analyzed using Monte Carlo simulation for a variety of scenarios: Four demand systems (Logit, Linear, Loglinear, and Almost ideal), two merger-specific, cost complementarity systems (Generalized Leontief and Quadratic), and a total of 40,000 mergers (8 scenarios, 5,000 mergers in each scenario). For each merger, we compute several measures of UPP (including standard and new), compute post-merger equilibrium, and compute effectiveness of UPP in terms of price-prediction accuracy and merger screening accuracy.

We find that with model-based cost efficiencies and using a more accurate first order approxi-

\footnotetext{
${ }^{1}$ The United Kingdom (2010) incorporates UPP to their horizontal merger assessment guidelines, $\S \S 5.4 .6$ - 5.4.11, highlighting the need to associate its analysis with price sensitivity of consumers through own and cross-price elasticities. In France(2013), as expressed in Les lignes directrices relatives au contrôle des concentrations V.D.2.c.(405-420), not only is UPP adopted, it highlights the need for proper efficiency estimates jointly with it. Brazil (2016) in Guia Análise de Atos de Concentração Horizontal §2.5.2. shows that the likelihood of harm from mergers with heterogeneous goods arises from the proximity of substitution (diversion).
} 
mation to compute UPP, there are substantial gains in prediction of post-merger equilibrium prices, as compared to current practice. In seven of eight scenarios, UPP based price predictions are within 3 percent of post-merger equilibrium prices at the median, both in absolute and relative terms. (In five scenarios, these are within 0.25 percent.)

Similarly, we find that with the new UPP measures, there are substantial gains in merger screening accuracy, as compared to current practice. In six of eight scenarios, UPP based merger screens (at 5\% price increase threshold) reduce total probability of false positives (flagging a merger for scrutiny incorrectly) and false negatives (letting a merger go through incorrectly) to less than 0.02. (In four scenarios, this is 0.007 or less.)

Finally, to provide a stricter comparison to existing practice, we compare the standard UPP formulation with higher ad hoc thresholds (of 5\%,10\%, and $15 \%$ price increase) to the new UPP measures with a $0 \%$ threshold. In every scenario, total probability of false positives and negatives is lower for the new UPP measures, and in most cases, there is a large reduction in the total error.

Our results support the use of UPP as a tool in antitrust analysis. In particular, effectiveness and accuracy of UPP is increased greatly by inclusion of model-based cost efficiencies.

The paper proceeds as follows. The next section includes a literature review. Section 3 details the theoretical framework and formulates different measures of UPP used in the analysis. Section 4 describes the Monte Carlo simulations and data generating process. Section 5 presents the results and analysis. Section 6 concludes.

\section{Literature Review}

"Antitrust is generally viewed as a public policy aimed at fostering a public good: that is, competition" (Buccirossi, 2008, p. xviii). Competition agencies ought to provide effective and timely evaluations of potential pro- and anti-competitive effects of proposed mergers. In the United States, Section 7 of the Clayton Act is the principal federal substantive law governing mergers, acquisitions, and joint ventures, and which deems unlawful the acquisition of a firm or its stocks such that "the effect of such acquisition may be substantially to lessen competition, or to tend to create a monopoly." Despite the clarity of the established goal, in reality things may not be as straightforward. Determining ex ante whether a merger will generate anticompetitive effects if allowed to go through requires a fair amount of analysis theoretical, empirical, and institutional.

Whenever screening potential mergers, the antitrust agencies attempt to disentangle and evaluate two potential sources of anticompetitive effects: unilateral and coordinated (Ivaldi et al., 2003; Werden, 2010). Unilateral effects arise whenever a newly merged firm engages in decision-making strategies characteristic of higher market power (in terms of ability to restrict output and price above the competitive level) due to consolidation. Coordinated effects, on the other hand, focus on changes in likelihood of collusion, either express or tacit, 
within a market due to consolidation. ${ }^{2}$ This paper focuses on unilateral effects, specifically those measuring incentive of consolidated firm to raise prices post-merger.

Since the development of the first merger guidelines in the United States in 1968 until its latest version in 2010, the focus has been to identify and guide the processes by which potential anticompetitive effects may arise. Shapiro (2010) reviews the main points of evolution and change over time, and highlights what has been learned in these four decades with respect to merger analysis. An overall trend is the shift in focus from narrower market concentration concerns to anticompetitive effects evaluated more holistically.

Market concentration methods, such as the Herfindahl Hirschman Index (HHI), continue to retain a prominent position in antitrust analysis. A well-known limitation of HHI or any other market-share based index for merger screening is the difficulty of market definition for natural competitors of differentiated goods (Werden \& Froeb, 1994, 1996; Hausman \& Leonard, 1996). This has fueled demand for alternative measures of anticompetitive effects arising from a merger.

Full merger simulations, an application of formal structural game-theoretical models to determine and predict unilateral anticompetitive effects, are expensive, time consuming, and depend on strong assumptions, prior beliefs and available data (Berry \& Pakes, 1993; Epstein \& Rubinfeld, 2001; Werden et al., 2003). Weinberg \& Hosken (2013) test the accuracy of merger simulation, and examine potential sources of differences in the simulated and directly estimated price effect. A substantial source of these differences are estimated to be changes in the cost structure - either a reduction or a slight augmentation in marginal costs. Budzinski \& Ruhmer (2009) show that merger simulations represent a major improvement in terms of merger screening due to its technical potential, nevertheless it should still be combined with alternative instruments of competition policy.

Due to the costly nature of full-merger simulations, it is useful to have alternative merger screening tools that are less expensive, quick, reliable, and theoretically grounded. UPP is being used increasingly in this regard as a pre-merger screening tool for horizontal mergers. $^{3}$

\section{UPP and First Order Approximation}

Farrell \& Shapiro (2010) develop upward pricing pressure as an index of likely unilateral effects from a merger, measured in monetary value of price increase resulting from a merger of horizontal competitors with partially differentiated goods. UPP indicates existence and strength of unilateral anticompetitive effects through an incentive to increase price of the goods produced by a merged firm. UPP doesn't claim to provide the exact amount that the merged firm will raise prices in post-merger equilibrium, but rather provides a measure of the initial incentive to do so, holding fixed other economic environment parameters, such as

\footnotetext{
${ }^{2}$ This change may manifest itself by the elimination of a maverick (i.e. a firm that seems to be a natural disruptor of tacit collusion, by constantly setting lower prices for example) or by simply reducing the number of players and facilitating that a collusive equilibrium might occur.

${ }^{3}$ See E.I. du Pont de Nemours \& Co., 353 U.S.at 592, City of NY v. Group Health Incorp., 649 F.3d 151 (2d Cir. 2011), FTC v. Lundbeck, Inc., 650 F.3d 1236 (8th Cir Aug 19, 2011)
} 
price and level of output of other firms, demand determinants, and so on. Therefore once the market re-equilibrates to a new post-merger equilibrium, the actual change in prices may be different from a change in first response.

This difference between first impulse to raise prices and post-merger equilibrium prices has been a source of debate in the literature. Schmalensee et al. (2009) prefer measures that predict post-merger equilibrium prices accurately, saying "hill's height is unrelated to how steep the hill is at its base." Farrell et al. (2011) point out that the first impulse has important information about final post-merger prices, saying "a ball that is kicked harder might not travel further [...] but as a general matter hard-kicked balls tend to go further."

Werden (1996) proposes a first-order approximation approach as an alternative to functional form simulation. Jaffe \& Weyl (2013) generalize the first-order approximation approach and show that it can be used to derive and improve the theoretical formulation of UPP. In particular, including a demand pass-through matrix makes the UPP computation more theoretically accurate as a first-impulse to raise prices. Their approach includes multi-product firms and is independent of particular functional forms for demand or costs. Willig (2011) investigates UPP computations in different directions, including how to consider pricing pressures in a merger that may alter the quality of products of merging firms. Moresi \& Salop (2013) study unilateral pricing incentives in vertical mergers taking under consideration cost efficiencies across upstream and downstream.

Miller et al. (2013a) investigate the accuracy of the first-order approximation in a Monte Carlo simulation of merger analysis in oligopoly models and compare it to the corresponding post-merger equilibrium. They find improvements in accuracy when using UPP with the first-order approximation. The employment of pass-through in merger simulation techniques (Hausman et al., 1994; Werden \& Froeb, 1994; Nevo, 2000) has been much studied in academic settings as well as employed by practitioners in a litigious setting. Miller et al. (2016) focuses on the role pass-through may play in improving the prediction of post merger prices.

Cheung (2016) evaluates the performance of UPP as a merger screening tool in contrast to standard structural merger simulation by generating hypothetical mergers using US airline industry data to feed into the model. She documents favorable results in "best case scenario" when full information is available, as well as within correct decile predictions. Garmon (2017) compares UPP with many other merger screening tools ${ }^{4}$ showing that "first-order pricing incentives of merged hospitals (in particular, WTP and UPP) are more accurate at flagging mergers that are potentially anticompetitive than the traditional tools of market definition and concentration measurement."

Miller et al. (2017) specifically compare results from UPP and first order approximation with other obtained through merger simulation for a variety of economic environments as well as different practitioner conditions (such as mis-observed demand elasticity, wrong functional form of demand and pass-through); and show that UPP is accurate with standard log-

\footnotetext{
${ }^{4}$ Merger screening tools utilized in Garmon (2017) include first order necessary conditions, such as diversion ratios and upward pricing pressure, willingness to pay (WTP); hospital merger simulations; and traditional concentration methods such as Herfindahl Hirschman Index (HHI).
} 
concave demand systems, slightly understating the effect in demands with greater convexity. Even more noticeable is that predicted errors with UPP do not exceed in magnitude those of merger simulation on misspecified simulation models, as well as in imprecise demand elasticities.

Jointly, these papers provide a compelling argument for adopting first order approximation techniques in merger analysis. They perform well as compared to full-blown merger simulations, are much less computationally heavy, and require less information under a variety of different scenarios. This strand of the UPP literature typically does not include efficiencies from a merger.

\section{Cost Efficiencies and UPP}

Efficiencies are often used as a motivation for mergers. Indeed, HMG (2010) state that "a primary benefit of mergers to the economy is their potential to generate significant efficiencies and thus enhance the merged firm's ability and incentive to compete, which may result in lower prices, improved quality, enhanced service, or new products." Moreover, "[i]n a unilateral effects context, incremental cost reductions may reduce or reverse any increases in the merged firm's incentive to elevate price" and thus, at least in principle, should be incorporated into post-merger price predictions relating to unilateral effects.

Nevertheless, these guidelines caution that efficiency claims alone are not enough to justify a merger, because "[e]ven when efficiencies generated through a merger enhance a firm's ability to compete, however, a merger may have other effects that may lessen competition and make the merger anticompetitive." Indeed, antitrust agencies are very skeptical of efficiency claims of pro-competitive effects in rule of reason analysis. ${ }^{6}$ In order to be considered seriously, efficiency claims by the merging parties have to be merger specific and verifiable. ${ }^{7}$ This has historically been interpreted to exclude most efficiency claims related to economies of scale, because scale economies can at least hypothetically be obtained through means other than a merger (Farrell \& Shapiro, 2001).

As mentioned above, efficiencies are not included in the standard UPP formulation. Indeed, in the standard formulation, the total cost of the merged firm is the sum of cost functions

\footnotetext{
${ }^{5}$ Horizontal Merger Guidelines (2010) §4

${ }^{6}$ For a comprehensive review of the historical evolution of antitrust policy regarding merger efficiency claims on both the United States and European Union, see (Gifford \& Kudrle, 2015, Chapter 3). Kinne (1999) explains in a little more detail specificities on the German case; Crane (2011) goes through the asymmetries and implicit bias of competition agencies both in the U.S. and European Union when it comes to the burden of proof and magnitude arising from potential mergers as opposed to figures regarding harm to competition.

7"The Agencies credit only those efficiencies likely to be accomplished with the proposed merger and unlikely to be accomplished in the absence of either the proposed merger or another means having comparable anticompetitive effects. These are termed merger-specific efficiencies[...] Efficiency claims will not be considered if they are vague, speculative, or otherwise cannot be verified by reasonable means.[...] Cognizable efficiencies are merger-specific efficiencies that have been verified and do not arise from anticompetitive reductions in output or service. Cognizable efficiencies are assessed net of costs produced by the merger or incurred in achieving those efficiencies." Department of Justice and Federal Trade Commission Horizontal Merger Guidelines (2010)
} 
of the merging firms, eliminating cross firm cost complementarities that typically form the basis of merger-specific efficiencies. As shown by Deneckere \& Davidson (1985), mergers in Bertrand-type markets with differentiated products yield higher prices in the absence of efficiencies.

Farrell \& Shapiro (2010) suggest accommodating efficiencies by including a "standard efficiencycredit", as in Warren-Boulton (1985), to serve as a proxy for merger-specific efficiencies. In the absence of a connection to the underlying model, merging firms typically argue for higher efficiency credits whereas antitrust agencies argue for lower numbers and require additional justification. Current practice is to arrive at an ad hoc adjustment. As mentioned in Simons \& Coate (2010), a limitation is that the "model would still lack empirical verification", and therefore, should not be used in lieu of merger-specific efficiencies.

We revisit the base model used to derive UPP and include merger-specific cost efficiencies in the model. Using the theoretical framework in Jaffe \& Weyl (2013), we include efficiencies in a tractable manner and derive the related UPP formulations. In our framework, cost efficiencies are made merger-specific by requiring these to be zero if output of either firm in the merger is zero. In other words, cost efficiencies are activated only for the merged firm and only when outputs of both merging firms are positive. The new formulations are naturally connected to existing formulations and show how to modify existing formulations to account for cost efficiencies in a transparent manner. Details are included in the next section.

\section{Theoretical Framework}

Following Jaffe \& Weyl (2013), let $I=\{1, \ldots, N\}$ be the set of $N \geq 2$ firms producing multiple products competing as Bertrand oligopolists with slightly differentiated goods. The quantity vector of each firm $i$ is given by $Q_{i}(P)$, where $P$ is the vector of all prices in the industry and $P_{i}$ is the component of $P$ with prices for goods of firm $i$. Profit for firm $i$ is given by $\pi_{i}=P_{i}^{\top} Q_{i}(P)-C_{i}\left(Q_{i}(P)\right)$, where $C_{i}$ is the cost function for firm $i$.

The standard UPP formulation is as follows. Suppose firms $i$ and $j$ merge. The profit maximization problem for the merged firm is given by

$$
\max \Pi_{M}=P_{i}^{\top} Q_{i}(P)+P_{j}^{\top} Q_{j}(P)-C_{i}\left(Q_{i}(P)\right)-C_{j}\left(Q_{j}(P)\right)
$$

The first order condition (with respect to $P_{i}$ ) may be written as:

$\tilde{h}_{i}(P) \equiv-\left(\frac{\partial Q_{i}(P)^{\top}}{\partial P_{i}}\right)^{-1} Q_{i}(P)-\left(P_{i}-\frac{\partial C_{i}}{\partial Q_{i}(P)}\right)+\left(\frac{\partial Q_{i}(P)^{\top}}{\partial P_{i}}\right)^{-1}\left(\frac{\partial Q_{j}(P)^{\top}}{\partial P_{i}}\right)\left(P_{j}-\frac{\partial C_{j}}{\partial Q_{j}(P)}\right)=0$

Comparing this to the first-order condition for firm $i$ pre-merger yields upward pricing pressure for good $i$. 


$$
U P P_{i}=\underbrace{-\left(\frac{\partial Q_{i}(P)^{\top}}{\partial P_{i}}\right)^{-1}\left(\frac{\partial Q_{j}(P)^{\top}}{\partial P_{i}}\right)}_{D_{i j}} \underbrace{\left(P_{j}-\frac{\partial C_{j}}{\partial Q_{j}}\right)}_{\left(P_{j}-M C_{j}\right)}
$$

This is the standard UPP formulation used widely in the literature and in antitrust practice. The term $-\left(\partial Q_{1}(P)^{\top} / \partial P_{1}\right)^{-1}\left(\partial Q_{2}(P)^{\top} / \partial P_{1}\right)$ is the diversion matrix, which measures proportion of sales lost by firm $i$ that are recaptured by firm $j$, and $\left(P_{2}-\partial C_{2} / \partial Q_{2}\right)$ is the margin for firm $j$. Both are evaluated at pre-merger values.

Notice that in this formulation there are no merger-specific cost efficiencies, because total cost for the merged firm is the sum of costs of the merging partners and there are no crossfirm cost complementarities. In order to distinguish this from other UPP calculations, we shall denote this standard formulation with no efficiencies as $U P P_{\text {NoEff }}$.

We include cross-firm cost complementarities by adding an interactive term in the profitmaximization problem of the merged firm as follows.

$$
\max \Pi_{M}=P_{i}^{\top} Q_{i}(P)+P_{j}^{\top} Q_{j}(P)-\left[C_{i}\left(Q_{i}(P)\right)+C_{j}\left(Q_{j}(P)\right)-\phi\left(Q_{i}(P), Q_{j}(P)\right)\right]
$$

The term $\phi\left(Q_{i}(P), Q_{j}(P)\right)$ is an adjustment (reduction) to total cost of the merged firm that depends on output of both firms. In order to capture merger-specific efficiencies, we require this term to be zero if output of either firm is zero: $\left.\phi\left(Q_{i}(P), 0\right)\right)=\phi\left(0, Q_{j}(P)\right)=0$.

The first-order condition for this problem is given by

$$
\begin{array}{r}
\tilde{h}_{i}(P)=-\left(\frac{\partial Q_{i}(P)^{T}}{\partial P_{i}}\right)^{-1} Q_{i}(P)-\left(P_{i}-\frac{\partial C_{i}}{\partial Q_{i}(P)}+\frac{\partial \phi\left(Q_{i}(P) ; Q_{j}(P)\right)}{\partial Q_{i}(P)}\right) \\
-\left(\frac{\partial Q_{i}(P)^{T}}{\partial P_{i}}\right)^{-1}\left(\frac{\partial Q_{j}(P)^{T}}{\partial P_{i}}\right)\left(P_{j}-\frac{\partial C_{j}}{\partial Q_{j}(P)}+\frac{\partial \phi\left(Q_{i}(P) ; Q_{j}(P)\right)}{\partial Q_{j}(P)}\right)
\end{array}
$$

Comparing this to the pre-merger first-order condition yields the following new UPP formulation.

$$
\widetilde{U P P}_{i}(P)=D_{i j}\left(P_{j}-M C_{j}\right)-\underbrace{\left(\frac{\partial \phi\left(Q_{i}(P) ; Q_{j}(P)\right)}{\partial Q_{i}(P)}\right)}_{\text {Efficiency } i}+D_{i j} \underbrace{\left(\frac{\partial \phi\left(Q_{i}(P) ; Q_{j}(P)\right)}{\partial Q_{j}(P)}\right)}_{\text {Efficiency } j}
$$

In this formulation, efficiencies show up in a tractable and intuitive manner. The term $D_{i j}\left(P_{j}-M C_{j}\right)$ is the standard UPP formulation. The term $\left(\partial \phi\left(Q_{i}(P) ; Q_{j}(P)\right) / \partial Q_{i}(P)\right)$ is an efficiency adjustment to the standard UPP formulation arising from own firm efficiency. It serves to lower upward pricing pressure for good $i$. The term $D_{i j}\left(\partial \phi\left(Q_{i}(P) ; Q_{j}(P)\right) / \partial Q_{j}(P)\right)$ is due to partner firm efficiency (modified by diversion matrix). It serves to increase upward 
pricing pressure for good $i$. The UPP formulation with efficiencies adjusts the standard UPP formulation for both these effects. In order to distinguish this from other UPP calculations, we shall denote this UPP formulation with model-based, merger-specific efficiencies as UPP ModEff.

As is well-known, the standard UPP formulation does not capture the full first-order effect for a merged firm to raise prices. As shown in Jaffe \& Weyl (2013), in order to get an accurate first order approximation of the impulse to raise prices post-merger, the UPP calculation should be modified by the post-merger pass through matrix. This translates into the following UPP formulation with first-order approximation.

$$
U P P_{F O A}=\left(\frac{\partial \tilde{h}}{\partial P}\left(P^{0}\right)\right)^{-1} \widetilde{U P P}
$$

Here, $\widetilde{h}$ is the first-order condition (listed above) for the merged firm and $\left(\partial \widetilde{h} / \partial P\left(P^{0}\right)\right)^{-1}$ and $\widetilde{U P P}$ are evaluated at pre-merger equilibrium prices. $U P P_{F O A}$ uses a theoretically accurate measure of the change in best response of the merged firm as compared to the firm pre-merger.

The next section implements these formulations in a Monte Carlo setting.

\section{Monte Carlo}

In order to estimate the effect of the theoretical framework with cost efficiencies on the postmerger equilibrium and different measures of UPP, we use different economic environments to simulate the model. We use four different demand formulations and two different cost formulations for a total of eight different scenarios.

For the demand side, we use four standard functional forms that have been used widely in academic research and merger analysis (Werden et al., 2003; Budzinski \& Ruhmer, 2009; Miller et al., 2013b). These are logit demand system, log-linear demand system, linear demand system, and almost ideal demand system. These are also used in other Monte Carlo studies of UPP (Miller et al., 2013a, 2016, 2017). Our demand calibration strategy follows Miller et al. (2017), as described in detail in their appendix. ${ }^{8}$

For the cost side, we use two functional forms used in the existing literature: generalized Leontief cost structure (Li \& Rosenman, 2001) and quadratic cost structure (Cohn et al., 1989; Martínez-Budría et al., 2003).

The multiple good generalized Leontief formulation is the following (Diewert, 1971; Hall, 1973; Caves et al., 1980):

$$
C(Q)=\sum_{i}^{m} \sum_{j}^{m} \alpha_{i j}\left(Q_{i} Q_{j}\right)^{\frac{1}{2}}
$$

\footnotetext{
${ }^{8}$ We are grateful to Professor Nathan Miller for sharing his code for this calibration.
} 
In the special case when firms $i$ and $j$ merge, and each produces one good, the cost function for the merged firm is given by:

$$
C\left(Q_{i}, Q_{j}\right)=\alpha_{i i} Q_{i}+\alpha_{j j} Q_{j}-\alpha_{i j} Q_{i}^{1 / 2} Q_{j}^{1 / 2}
$$

In this case, the interactive term is $\phi\left(Q_{i}, Q_{j}\right)=\alpha_{i j} Q_{i}^{1 / 2} Q_{j}^{1 / 2}$ and it satisfies merger-specific cross complementarity that cannot be realized apart from consolidation; $\phi\left(Q_{i}, 0\right)=\phi\left(0, Q_{j}\right)=$ 0 . Notice that

$$
\begin{aligned}
& \Delta M C_{i}=\frac{\partial \phi\left(Q_{i} ; Q_{j}\right)}{\partial Q_{i}}=.5 \alpha_{i j}\left(\frac{Q_{j}}{Q_{i}}\right)^{1 / 2} \\
& \Delta M C_{j}=\frac{\partial \phi\left(Q_{i} ; Q_{j}\right)}{\partial Q_{j}}=5 \alpha_{i j}\left(\frac{Q_{i}}{Q_{j}}\right)^{1 / 2}
\end{aligned}
$$

The multiple good quadratic formulation is the following (Caves et al., 1980):

$$
C(Q)=\sum_{i}^{m} Q_{i}^{2}+\frac{1}{2} \sum_{i}^{m} \sum_{j \neq i}^{m} \beta_{i i} Q_{i} Q_{j}
$$

In the special case when firms $i$ and $j$ merge, and each produces one good, the cost function for the merged firm is given by:

$$
C\left(Q_{i}, Q_{j}\right)=\beta_{i i} Q_{i}^{2}+\beta_{j j} Q_{j}^{2}-\beta_{i j} Q_{i} Q_{j}
$$

In this case, the interactive term is $\phi\left(Q_{i}, Q_{j}\right)=\beta_{i j} Q_{i} Q_{j}$ and it also satisfies merger-specific cross complementarity that is activated only from a merger, in the sense that $\phi\left(Q_{i}, 0\right)=$ $\phi\left(0, Q_{j}\right)=0$. Notice that

$$
\begin{aligned}
& \Delta M C_{i}=\frac{\partial \phi\left(Q_{i} ; Q_{j}\right)}{\partial Q_{i}}=\beta_{i j} Q_{j} \\
& \Delta M C_{j}=\frac{\partial \phi\left(Q_{i} ; Q_{j}\right)}{\partial Q_{j}}=\beta_{i j} Q_{i}
\end{aligned}
$$

The data generating process is the following.

We suppose that each industry contains four firms competing in prices with slightly differentiated goods. Each firm produces a single output and industry equilibrium is BertrandNash.

1. Market shares are randomly drawn for each of the four firms and an outside good. The actual market shares that are used in the process are normalized to aggregate to one for the market in question. The margin for the first firm is randomly drawn with support $[0.2,0.8]$. 
2. The parameters for the interactive term in the cost structures are randomly drawn with support $[0,1] .^{9}$

3. Given the market shares and margins, it is possible to calibrate a logit demand system; and thus demand elasticities as of the pre-merger equilibrium. Notice that the demand system is such that its parameters are chosen to rationalize the drawn data on the previous steps. In this study, consumer substitution behavior is proportional to market shares. These parameters are identified exactly given market shares, prices, and a single margin.

4. Once the logit demand system is obtained, it is possible to calibrate the remaining demand functional forms (Log-Linear, Linear and Almost Ideal) such that they are compatible with the logit demand elasticities. Similarly to the logit case, the demand systems' parameters are perfectly identified given market shares, prices, and logit demand elasticities.

5. In each scenario, two firms go through a merger. Post-merger equilibrium prices are computed as well as various measures of upward pricing pressure and first order approximation.

6. Repeat these steps until 5,000 draws of data are obtained.

This process yields a total of 40,000 mergers (8 scenarios with 5,000 mergers each).

In order to analyze the accuracy of UPP for price prediction and for merger screening, we use the following four measures. Details for each are given below.

- UPP $P_{\text {NoEff }}$ - This is the standard and widely used UPP calculation with no efficiencies. It serves as a baseline.

- UPP AvgEff - The is the standard UPP calculation adjusted for average merger efficiencies. It serves as a benchmark for current practice.

- UPP $P_{\text {ModEff }}$ - This is UPP with model-based, merger-specific cost efficiencies, as derived above.

- UPP $P_{F O A}$ - This is UPP with model-based efficiencies and first-order approximation, as derived above.

A starting point for UPP calculations is the standard UPP calculation measuring the value of diverted sales.

$$
U P P_{N o E f f}=U P P_{i}=D_{i j}\left(P_{j}-M C_{j}\right)
$$

As discussed above, $U P P_{N o E f f}$ does not include cost efficiencies. This serves as a baseline for additional analysis.

\footnotetext{
${ }^{9}$ The rationale behind the support of these parameters being non-negative is as follows: If the firms would be more inefficient operating jointly than separately, then even if they merge, there is reason enough to believe it would continue operations disjointly.
} 
The second measure we use is the value of diverted sales adjusted for average merger efficiencies.

$$
U P P_{\text {AvgEff }}=D_{i j}\left(P_{j}-M C_{j}\right)-\underbrace{-\underbrace{\overline{\left(\Delta M C_{i}\right)}}_{\text {Own }}+D_{i j} \overline{\left(\Delta M C_{j}\right)}}_{\text {Both }}
$$

This measure is an estimate for current practice in the following sense. It is well-known that in the absence of cost efficiencies, UPP tends to overestimate the increase in post-merger prices. The standard current practice to account for this is to lower the UPP computation by some amount, motivating it as a reduction due to cost efficiencies. The amount of this reduction is a frequent source of debate. When UPP computation is high, merging parties argue with antitrust agencies that the UPP calculation should be lowered significantly, because there are cost efficiencies from the merger, but antitrust agencies argue for more realistic numbers and require additional justification. Current practice is to arrive at an ad hoc adjustment, sometimes termed an efficiency credit.

The measure $U P P_{\text {AvgEff }}$ is a benchmark for the current practice of efficiency credits, in the sense that it adjusts baseline UPP calculation $U P P_{N o E f f}$ for the average efficiency realized under a particular cost complementarity structure. In other words, in the absence of modeling cost efficiencies, if merging parties and antitrust agencies have to agree to an efficiency credit, their best guess would be the efficiency that a particular technology generates on average, yielding the measure $U P P_{\text {AvgEff }}$.

The third measure we use is the UPP calculation adjusted for model-based and mergerspecific cost efficiencies. This is what the standard UPP calculation would be if we derived it using model-based cost complementarities.

$$
U P P_{\text {ModEff }}=D_{i j}\left(P_{j}-M C_{j}\right)-\underbrace{-\underbrace{\left(\Delta M C_{i}\right)}_{\text {Own }}+D_{i j}\left(\Delta M C_{j}\right)}_{\text {Both }}
$$

The final measure we use adjusts the UPP computation with model-based cost efficiencies by the pass-through matrix.

$$
U P P_{F O A}=\left(\frac{\partial \tilde{h}}{\partial P}\left(P^{0}\right)\right)^{-1}(D_{i j}\left(P_{j}-M C_{j}\right) \underbrace{-\underbrace{\left(\Delta M C_{i}\right)}_{\text {Own }}+D_{i j}\left(\Delta M C_{j}\right)}_{\text {Both }})
$$

Here, $\widetilde{h}$ is the first-order condition for the merged firm and $\left(\partial \widetilde{h} / \partial P\left(P^{0}\right)\right)^{-1}$ is the post-merger pass-through matrix. $U P P_{F O A}$ is a theoretically more accurate measure of the first impulse to change prices. As usual, to operationalize this, the post-merger pass-through matrix is evaluated at pre-merger equilibrium values. 
For each merger, we compute these four UPP measures and compare them with the postmerger equilibrium price.

The next section shows the results of the calibration and simulation process; as well as measures of accuracy of prediction and decision-making errors in merger screening for each of the methods described above.

\section{$5 \quad$ Results}

Table 1 presents some descriptive summary statistics for the data generated using Monte Carlo. The median market share for firms is $20 \%$, which is consistent with drawing market shares for four firms and an outside good. Eight percent of the margins are distributed in between 0.247 and 0.746 with median at 0.471 (these are pre-merger margin values, no including the merger-efficiencies).

Market concentrations, as measured by Herfindahl Hirschman Index, has a median premerger of 1981, considered to be a moderately concentrated market according to the Horizontal Merger Guidelines (2010). ${ }^{10}$ Already at the $10^{\text {th }}$ percentile, markets are at least moderately concentrated, whereas at the $90^{\text {th }}$ percentile markets are already highly concentrated pre-merger. This is consistent with a market comprised of four firms. ${ }^{11}$ Market concentration post-merger, also measured through HHI has a median of 2706, a highly concentrated market. Eighty percent of the markets are in between 1795 and 4066. This increase in concentration is consistent with a market reduced to three firms. $\triangle H H I$ has median at 654, which would trigger further scrutiny from the Agencies. ${ }^{12}$ Figure 26 shows the density kernels for HHI's pre and post-merger for all mergers.

Different measures of UPP yield different post-merger price predictions. $U P P_{N o E f f}$ results in a median of $10.7 \%$ increase in in prices, and eight percent distribution between $2.1 \%$ and $27.5 \%$. The medians for post-merger price increase prediction diminish as more robust methods of incorporating merger-efficiencies are applied, culminating at $U P P_{F O A}$ with median $1.2 \%$ and eighth percent distributed between $-61.9 \%$ and $25.3 \%$.

Own merger pass through is highest at Log-Linear and lowest at Linear demands. Crossmerger pass-through are highest at Almost Ideal and lowest at Log-Linear demand. Merger price effects are smallest with Linear, with Median at 1.7\%, Logit with $2.7 \%$, Almost Ideal at $5 \%$ and Log-Linear at $7.8 \%$.

\section{Price Prediction Accuracy}

For price prediction accuracy, we compute absolute errors and relative errors as follows.

\footnotetext{
${ }^{10}$ According to the U.S. Department of Justice and Federal Trade Commission Horizontal Merger Guidelines (2010), §5, a market is considered unconcentrated if $H H I \leq 1500$, moderately concentrated if $1500<H H I \leq 2500$ and highly concentrated if $H H I>2500$.

${ }^{11} \mathrm{~A}$ market with four equal sized firms would be on the threshold between moderately and highly concentrated.

${ }^{12}$ According to HMG(2010) §5, mergers that increase HHI by less than 100 are unlikely to be challenged, whereas mergers that increase it by more than 200 will likely require further action.
} 
Table 1: Descriptive Statistics

\begin{tabular}{|c|c|c|c|c|c|}
\hline & Median & $10 \%$ & $25 \%$ & $75 \%$ & $90 \%$ \\
\hline \multicolumn{6}{|c|}{ MARKET CONDITIONS } \\
\hline Market share & 0.201 & 0.050 & 0.114 & 0.276 & 0.339 \\
\hline Margin & 0.471 & 0.247 & 0.333 & 0.623 & 0.746 \\
\hline \multicolumn{6}{|c|}{ Market Concentration } \\
\hline Pre-Merger & 1981 & 1418 & 1642 & 2436 & 2872 \\
\hline Post-Merger & 2706 & 1795 & 2159 & 3360 & 4066 \\
\hline$\triangle H H I$ & 654 & 113 & 310 & 1075 & 1527 \\
\hline \multicolumn{6}{|c|}{ Upward Pricing Pressure } \\
\hline$U P P_{N o E f f}$ & 0.107 & 0.021 & 0.053 & 0.184 & 0.265 \\
\hline$U P P_{\text {AvgEff }}$ & 0.021 & -0.115 & -0.045 & 0.104 & 0.192 \\
\hline$U P P_{M o d E f f}$ & 0.013 & -0.248 & -0.070 & 0.058 & 0.108 \\
\hline$U P P_{F O A}$ & 0.012 & -0.619 & -0.115 & 0.074 & 0.253 \\
\hline \multicolumn{6}{|c|}{ OWn MERger PASS-Through } \\
\hline Logit & 0.801 & 0.675 & 0.733 & 0.880 & 0.943 \\
\hline Linear & 0.533 & 0.510 & 0.520 & 0.550 & 0.569 \\
\hline Log-Linear & 1.927 & 1.344 & 1.515 & 2.671 & 3.601 \\
\hline Almost Ideal & 1.210 & 0.782 & 0.907 & 1.776 & 2.522 \\
\hline \multicolumn{6}{|c|}{ Cross Merger Pass-Through } \\
\hline Logit & 0.038 & 0.007 & 0.018 & 0.063 & 0.089 \\
\hline Linear & 0.090 & 0.024 & 0.053 & 0.120 & 0.152 \\
\hline Log-Linear & 0.000 & -0.000 & -0.000 & 0.000 & 0.000 \\
\hline Almost Ideal & 0.227 & 0.057 & 0.125 & 0.409 & 0.778 \\
\hline \multicolumn{6}{|c|}{ Merger Price Effects } \\
\hline Logit & 0.023 & -0.158 & -0.019 & 0.064 & 0.113 \\
\hline Linear & 0.017 & -0.137 & -0.038 & 0.054 & 0.106 \\
\hline Log-Linear & 0.078 & -0.444 & -0.047 & 0.288 & 0.939 \\
\hline Almost Ideal & 0.050 & -0.287 & -0.025 & 0.175 & 0.503 \\
\hline
\end{tabular}

Notes: Summary statistics are based on 5,000 randomly-drawn sets of data on the pre and post-merger equilibria.The values for market share and margin are for all four firms. Market share and margin are drawn randomly in the data generating process. Own merger pass-through is the first element of the diagonal of $\left[\widetilde{h}(P) / \partial P\left(P^{0}\right)\right]^{-1}$, and cross merger pass-through is the first off-diagonal element of $\left[\widetilde{h}(P) / \partial P\left(P^{0}\right)\right]^{-1}$. The merger price effects are the change in firm 1's equilibrium price. 

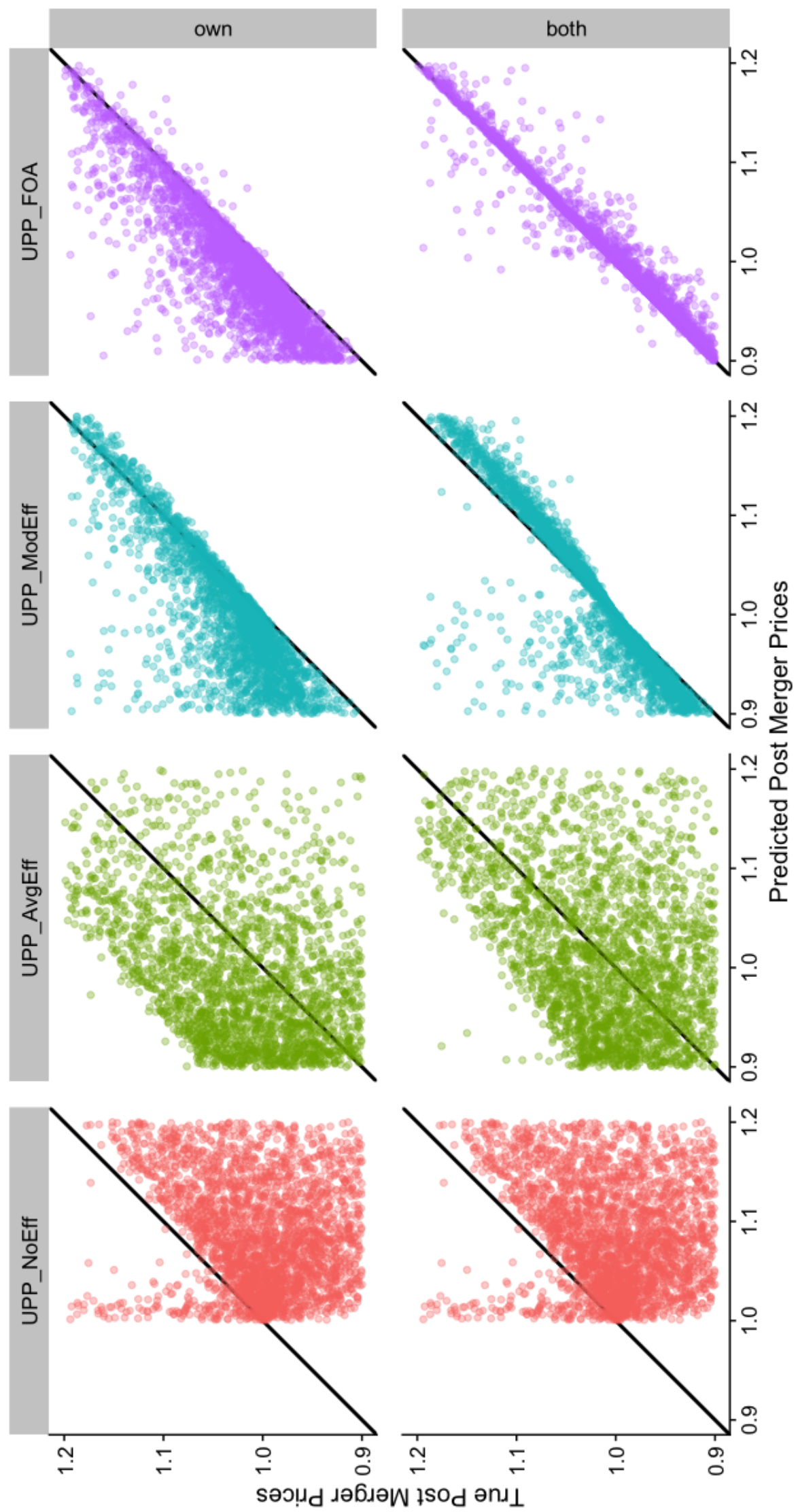

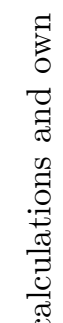

客

0
0
0

吕晋

$\stackrel{0}{-}$

范

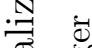

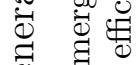

$v$ in

च.

苟 总

ค

.

$\mapsto$ 菏

- 010

苞

$\overbrace{-1}$

ᄃ.

फ

仓्]

:

导峦

긍 छ्ञ

$\stackrel{4}{\sharp}$

$.00 \frac{9}{1}$

胥

?․ำ

荡

․․․

क

$\frac{6}{n}$

进

步 
Absolute prediction error: $A P E=\left|P_{U P P}-P_{P o s t}\right|$

Relative prediction error: $R P E=\left|P_{U P P}-P_{\text {Post }}\right| / P_{\text {Post }}$

Here $P_{U P P}$ is the price given by a particular UPP calculation and $P_{\text {Post }}$ is the computed postmerger equilibrium price. As pre-merger prices are normalized to unity, APE gives prediction error in percentage points and RPE gives percent error. For example, if $P_{U P P}=1.11$ and $P_{\text {Post }}=1.05$ then (because pre-merger price is 1 ), $A P E=6$ percentage points and $R P E=4.5$ percent.

Figure 1 presents the analysis for the environment with logit demand system and generalized Leontief cost structure. The figure has eight panels. The four columns correspond to the four UPP calculations defined above: $U P P_{\text {NoEff }}, U P P_{\text {Avgeff }}, U P P_{\text {ModEff }}$, and $U P P_{F O A}$. The top row corresponds to the case where these calculations are made using own firm efficiency only (a case that is frequently used in practice) and the bottom row corresponds to the case where these calculations are made using both own firm efficiency and partner firm efficiency. The bottom row uses the four calculations defined above.

In each panel, the x-axis measures the predicted post-merger price using a particular UPP calculation, and the $\mathrm{y}$-axis measures the true post-merger equilibrium price. Each point in a panel corresponds to one merger. Points on the diagonal are those mergers for which the price prediction using the UPP calculation for that panel is exactly the same as the true post-merger equilibrium price. Points above the diagonal are those mergers for which the UPP calculation under-predicts the true post-merger price. Points below the diagonal are those mergers for which the UPP calculation over-predicts the true post-merger price.

Consider the first column in Figure 1. In the top panel (labeled $U P P_{N o E f f}$ ), the x-axis measures the post-merger price increase using $U P P_{N o E f f}$ and the y-axis measures the true post-merger equilibrium price. As $U P P_{\text {NoEff }}$ excludes efficiencies, most of the data overpredicts the true post-merger prices and lies below the diagonal, as expected. The data appear truncated at 1.0 (the pre-merger equilibrium price) because in the absence of cost efficiencies, $U P P_{N o E f f}$ predicts a price increase, even when the true post-merger price is lower, as expected. The bottom panel is the same as the top panel, because the difference in UPP calculation between own firm efficiency and the combined efficiency of both firms arises only when the UPP calculation includes efficiencies. In both panels, median APE is 14.3 p.p. and median RPE is $13.6 \%$. The density kernels of APE are given in the corresponding panels in Figure 2 and that of RPE in the corresponding panels in Figure 3.

The second column in Figure 1 (labeled $U P P_{\text {AvgEff }}$ ) adjusts $U P P_{N o E f f}$ for an efficiency credit based on the average efficiency generated by a particular technology (Generalized Leontief in this case). As discussed above, this a proxy for the current practice of adjusting the UPP calculation for an efficiency credit. As compared to panels in column 1, this moves the data toward the left. The top panel in this column considers average efficiency for own firm only and the bottom panel considers average combined efficiency for both partners in a merger. As compared to the first column, the data in the second column is dispersed somewhat more evenly across the diagonal, indicating improved price prediction accuracy. This shows up in lower price prediction errors. In the top panel, median APE is 10.7 p.p. (a gain in price prediction accuracy of about 3.6 percentage points over $\left.U P P_{N o E f f}\right)$ and median 


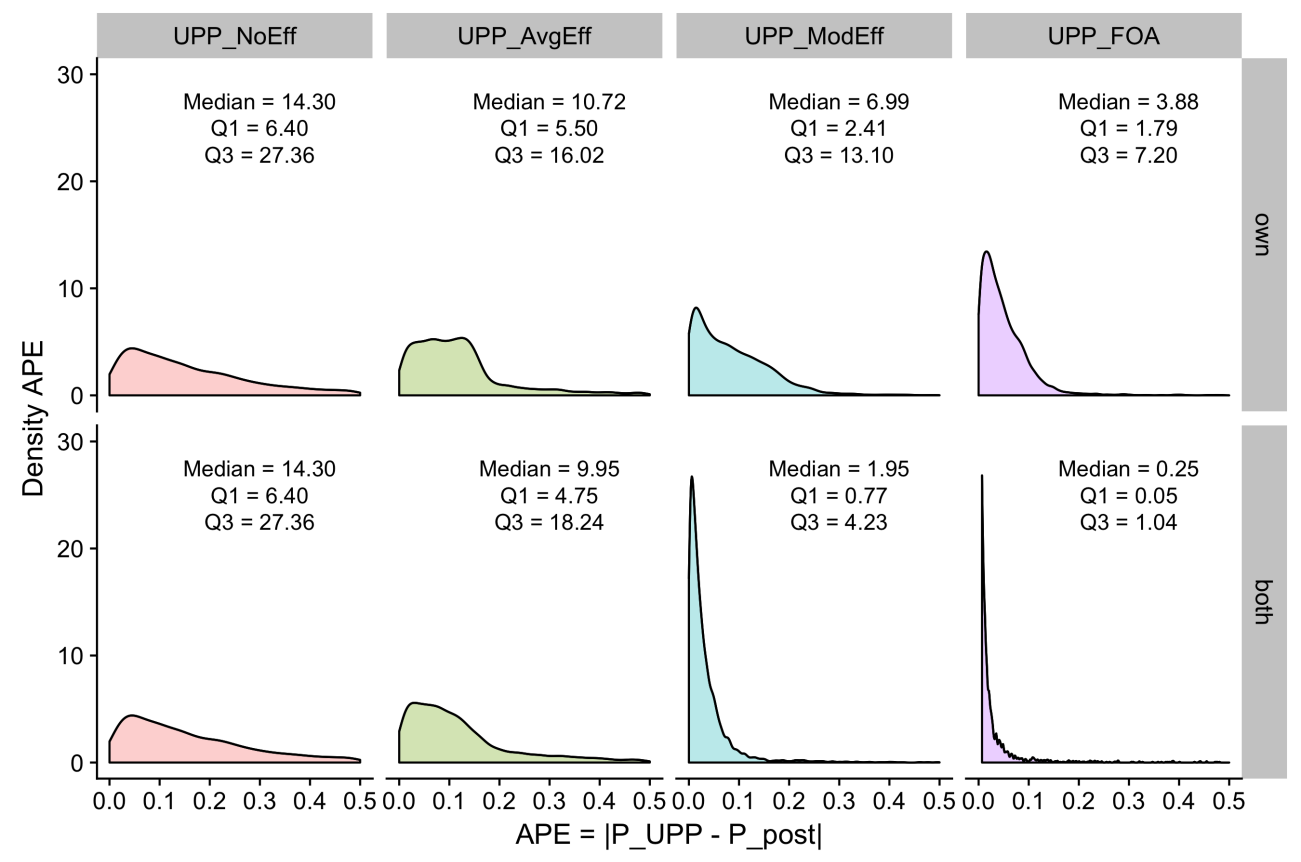

Figure 2: Absolute Prediction Errors - Logit Demand System, Generalized Leontief Cost Portrays density kernels for absolute prediction errors, as well as the median absolute prediction error, first and third quartile for each specification.

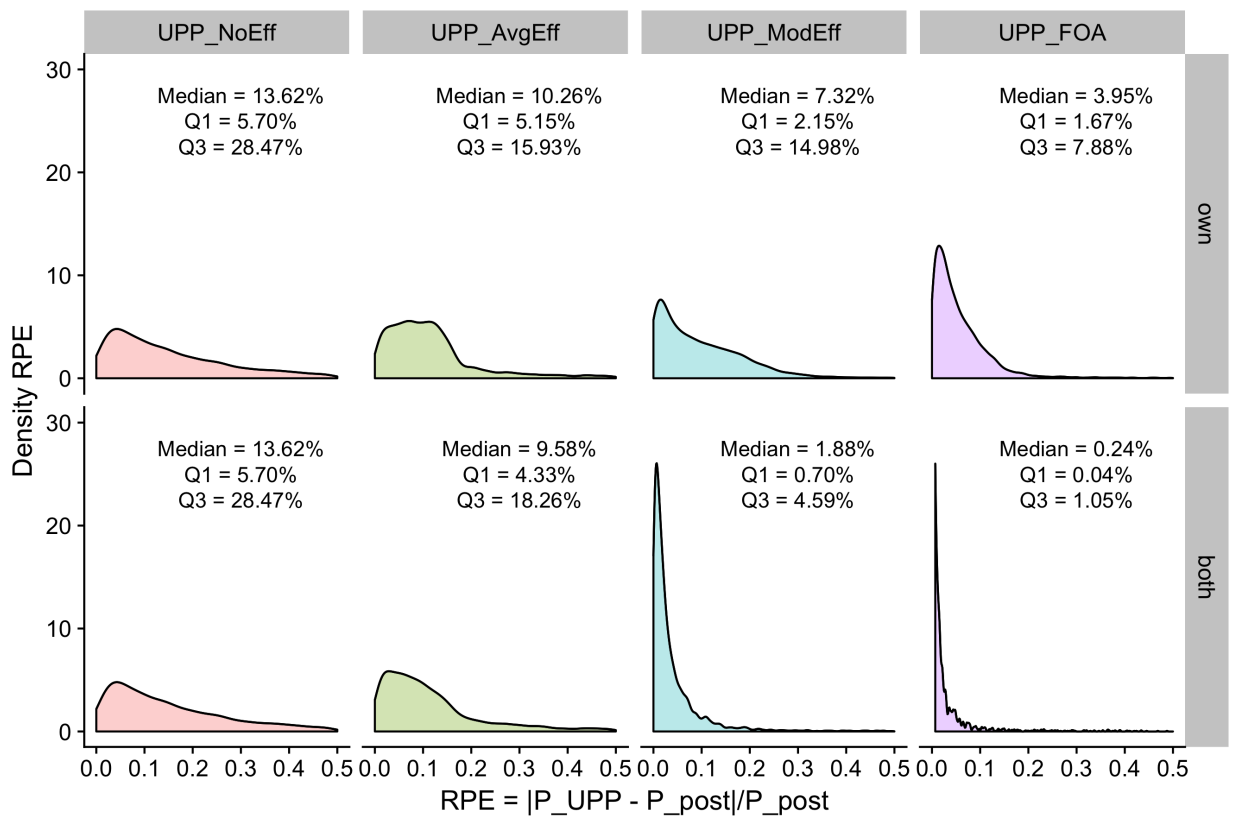

Figure 3: Relative Prediction Errors - Logit Demand System, Generalized Leontief Cost

Portrays density kernels for relative prediction errors, as well as the median relative prediction error, first and third quartile for each specification. 
RPE is $10.3 \%$ (a gain of about 3.3 percentage points over $U P P_{N o E f f}$ ). In the bottom panel, the corresponding numbers are 9.9 p.p. for APE (a gain of 4.4 p.p.) and $9.6 \%$ for RPE (a gain of 4 p.p.). The density kernels of APE are given in the corresponding panels in Figure 2 and that of RPE in the corresponding panels in Figure 3.

The third column in Figure 1 (labeled $U P P_{\text {Modeff }}$ ) uses the new UPP calculation based on model-based, merger-specific generalized Leontief cost complementarities. Both panels show a noticeable clustering of the data around the diagonal, indicating further improvements in price prediction. In the top panel, median APE is 7 p.p. (a gain of 3.7 percentage points over current practice proxy using $U P P_{\text {AvgEff }}$ ) and median RPE is $7.3 \%$ (a gain of about 3.3 percentage points over $U P P_{\text {AvgEff }}$ ). The bottom panel shows that accounting for the combined efficiency effect of both merger partners yields even greater gains in price prediction accuracy, consistent with the theory. In the bottom panel, median APE is 2 p.p. (a gain of 7.9 p.p. over $U P P_{\text {AvgEff }}$ ) and median RPE is $1.9 \%$ (a gain of 7.7 p.p.). The density kernels of APE are given in the corresponding panels in Figure 2 and that of RPE in the corresponding panels in Figure 3.

Finally, the fourth column in Figure 1 (labeled $U P P_{F O A}$ ) uses the first-order approximation to adjust $U P P_{M o d E f f}$ by the pass-through matrix. As mentioned above, this is a theoretically accurate measure of the first impulse to change prices. Both panels show greater clustering of data around the diagonal, with notable improvement in the bottom panel. In the top panel, median APE shrinks to 3.9 p.p. (a gain of 6.8 percentage points over current practice proxy using $U P P_{\text {AvgEff }}$ ) and median RPE is $4 \%$ (a gain of about 7.3 percentage points over $U P P_{\text {AvgEff }}$ ). In the bottom panel, median APE is only 0.3 p.p. (a gain of 9.6 p.p. over $U P P_{\text {AvgEff }}$ ) and median RPE is $0.2 \%$ (a gain of 9.4 p.p.).

Put differently, in the bottom panel of column four, absolute price prediction errors decrease $97 \%$ (from 9.9 p.p. to 0.3 p.p., at the median) and relative price predictions errors decrease $97 \%$ (from $9.6 \%$ to $0.2 \%$, at the median) as we move from current practice (using $U P P_{\text {AvgEff }}$ ) to a more theoretically accurate measure using $U P P_{F O A}$. More generally, the entire density kernel of the corresponding APE (Figure 2, bottom right panel) and of RPE (Figure 3, bottom right panel) compresses toward zero.

Figures 1, 2, and 3 indicate presence of substantial gains from reforming the standard UPP calculation to include model-based cost efficiencies (for both merging partners) and firstorder approximation. These results are based on Logit demand and Generalized Leontief costs. A similar pattern is seen for the other seven scenarios as well. This is documented in figures 5-25 in the appendix.

A summary of all eight scenarios is given in Table 2. As shown in Table 2, in seven of eight scenarios, $U P P_{F O A}$ based price predictions are within $3 \%$ of post-merger equilibrium prices at the median, both in absolute and relative terms (The log-linear case is an exception, likely related to curvature of utility, causing the diagonal elements of the merger pass-through matrix to exceed one, as documented in Miller et al. (2017)). The average reduction in price prediction errors $\left(U P P_{F O A}\right.$ compared to $\left.U P P_{A v g E f f}\right)$ in these seven scenarios is $93 \%$.

Moreover, in five scenarios, $U P P_{F O A}$ based price predictions are within $0.25 \%$ of post-merger equilibrium prices at the median, both in absolute and relative terms. The average reduction 
Table 2: Improvement in Price Prediction

\begin{tabular}{|c|c|c|c|c|c|c|c|c|c|}
\hline \multicolumn{10}{|c|}{ Generalized LEONTIEF COST } \\
\hline \multirow[t]{2}{*}{ Logit Demand } & NoEff & AvgEff & ModEff & FOA & Linear Demand & NoEff & AvgEff & ModEff & FOA \\
\hline & \multicolumn{4}{|c|}{ APE (p.p.) } & & \multicolumn{4}{|c|}{ APE (p.p.) } \\
\hline Median & 14.30 & 9.95 & 1.95 & 0.25 & Median & 14.34 & 8.73 & 2.26 & 0.00 \\
\hline Absolute Gain over AvgEff & & & 8.00 & 9.69 & Absolute Gain over AvgEff & & & 6.47 & 8.73 \\
\hline \multirow[t]{2}{*}{ Relative Gain over AvgEff } & & & 80.39 & 97.47 & Relative Gain over AvgEff & & & 74.08 & 100.00 \\
\hline & \multicolumn{4}{|c|}{$\operatorname{RPE}(\%)$} & & \multicolumn{4}{|c|}{$\mathrm{RPE}(\%)$} \\
\hline Median & 13.62 & 9.58 & 1.88 & 0.24 & Median & 14.38 & 8.72 & 2.23 & 0.00 \\
\hline Absolute Gain over AvgEff & & & 7.70 & 9.34 & Absolute Gain over AvgEff & & & 6.50 & 8.72 \\
\hline Relative Gain over AvgEff & & & 80.33 & 97.51 & Relative Gain over AvgEff & & & 74.49 & 100.00 \\
\hline \multirow[t]{2}{*}{ Log-Linear Demand } & NoEff & AvgEff & ModEff & FOA & Almost Ideal Demand & NoEff & AvgEff & ModEff & FOA \\
\hline & \multicolumn{4}{|c|}{ APE (p.p.) } & & \multicolumn{4}{|c|}{ APE (p.p.) } \\
\hline Median & 33.15 & 27.79 & 19.55 & 15.18 & Median & 21.43 & 16.71 & 8.17 & 1.71 \\
\hline Absolute Gain over AvgEff & & & 8.24 & 12.60 & Absolute Gain over AvgEff & & & 8.54 & 14.99 \\
\hline \multirow[t]{2}{*}{ Relative Gain over AvgEff } & & & 29.64 & 45.36 & Relative Gain over AvgEff & & & 51.10 & 89.74 \\
\hline & \multicolumn{4}{|c|}{$\operatorname{RPE}(\%)$} & & \multicolumn{4}{|c|}{$\operatorname{RPE}(\%)$} \\
\hline Median & 34.75 & 28.79 & 22.92 & 16.04 & Median & 22.01 & 16.93 & 9.15 & 1.76 \\
\hline Absolute Gain over AvgEff & & & 5.87 & 12.75 & Absolute Gain over AvgEff & & & 7.78 & 15.17 \\
\hline ver AvgEff & & & 20.40 & 44.27 & Relative Gain over AvgEff & & & 45.94 & 89.60 \\
\hline \multicolumn{10}{|c|}{ Quadratic Cost } \\
\hline \multirow[t]{2}{*}{ Logit Demand } & NoEff & AvgEff & ModEff & FOA & Linear Demand & NoEff & AvgEff & ModEff & FOA \\
\hline & \multicolumn{4}{|c|}{ APE (p.p.) } & & \multicolumn{4}{|c|}{ APE (p.p.) } \\
\hline Median & 5.89 & 3.84 & 0.62 & 0.02 & Median & 6.15 & 3.94 & 1.12 & 0.00 \\
\hline Absolute Gain over AvgEff & & & 3.22 & 3.82 & Absolute Gain over AvgEff & & & 2.83 & 3.94 \\
\hline \multirow[t]{2}{*}{ Relative Gain over AvgEff } & & & 83.81 & 99.47 & Relative Gain over AvgEff & & & 71.67 & 100.00 \\
\hline & \multicolumn{4}{|c|}{$\operatorname{RPE}(\%)$} & & \multicolumn{4}{|c|}{$\mathrm{RPE}(\%)$} \\
\hline Median & 5.63 & 3.73 & 0.59 & 0.02 & Median & 5.91 & 3.82 & 1.08 & 0.00 \\
\hline Absolute Gain over AvgEff & & & 3.14 & 3.71 & Absolute Gain over AvgEff & & & 2.75 & 3.82 \\
\hline Relative Gain over AvgEff & & & 84.16 & 99.49 & Relative Gain over AvgEff & & & 71.86 & 100.00 \\
\hline \multirow[t]{2}{*}{ Log-Linear Demand } & NoEff & AvgEff & ModEff & FOA & Almost Ideal Demand & NoEff & AvgEff & ModEff & FOA \\
\hline & \multicolumn{4}{|c|}{ APE (p.p.) } & & \multicolumn{4}{|c|}{ APE (p.p.) } \\
\hline Median & 5.37 & 8.73 & 9.60 & 2.92 & Median & 2.61 & 4.30 & 3.60 & 0.21 \\
\hline Absolute Gain over AvgEff & & & -0.87 & 5.81 & Absolute Gain over AvgEff & & & 0.70 & 4.08 \\
\hline \multirow[t]{2}{*}{ Relative Gain over AvgEff } & & & -9.98 & 66.54 & Relative Gain over AvgEff & & & 16.27 & 95.00 \\
\hline & \multicolumn{4}{|c|}{$\operatorname{RPE}(\%)$} & & & $\mathrm{RPH}$ & $(\%)$ & \\
\hline Median & 4.71 & 7.68 & 8.32 & 2.29 & Median & 2.36 & 4.11 & 3.33 & 0.20 \\
\hline Absolute Gain over AvgEff & & & -0.64 & 5.39 & Absolute Gain over AvgEff & & & 0.78 & 3.91 \\
\hline Relative Gain over AvgEff & & & -8.38 & 70.15 & Relative Gain over AvgEff & & & 18.92 & 95.21 \\
\hline
\end{tabular}


in price prediction errors $\left(U P P_{F O A}\right.$ compared to $\left.U P P_{A v g E f f}\right)$ in these five scenarios is $98 \%$. Altogether, the results show considerable evidence for using model-based efficiencies and a more accurate first-order approximation in UPP calculations.

\section{Merger Screening Accuracy}

We also use these data to investigate accuracy of different UPP formulations as pre-merger screening tools. As mentioned earlier, UPP is being used increasingly as a pre-merger screening tool by antitrust agencies in the United States and worldwide, mainly because it is relatively quick and easy to implement, requires less information than some other measures, and is grounded in theory.

The typical use of UPP is to flag a merger for further scrutiny if the UPP calculation is above a given threshold. As UPP is not a perfect predictor of post-merger prices, this leads to two familiar errors: false positives and false negatives.

A false positive occurs when the UPP screen flags a merger for further analysis but postmerger equilibrium prices are below the acceptable threshold. A false positive may lead to unnecessary use of resources by both the antitrust agencies and the merging parties to investigate or block a merger that does not have significant anticompetitive effects. We term this a Type I error.

A false negative occurs when the UPP screen does not flag a merger for further analysis but post-merger equilibrium prices are above the acceptable threshold. A false negative allows a merger to go through even if it has significant anticompetitive effects and may harm consumers. We term this a Type II error.

As a baseline, consider a $5 \%$ price increase threshold. This is a common threshold in antitrust analysis, and is also used in the SSNIP test.

Graphically, in each panel in Figure 4, draw a vertical line intersecting the x-axis at 1.05, and a horizontal line intersecting the y-axis at 1.05. Mergers to the right of the vertical line are flagged for further scrutiny by the UPP screen and mergers to the left are not. Mergers above the horizontal line have high post-merger price increases (relative to the acceptable threshold) and mergers below the line have low post-merger price increases. Therefore, all mergers in the bottom right quadrant are false positives (flagged for further scrutiny incorrectly) and all mergers in the top left quadrant are false negatives (letting a merger go through incorrectly).

As expected, and as shown in the first column in Figure 4 (labeled $U P P_{\text {NoEff }}$ ), in the presence of merger efficiencies, not including these efficiencies in UPP calculation leads to a sizable number of false positives (about 57.9 percent of all mergers) and perhaps a few false negatives (3.5 percent). In this case, the total probability of making a type I or type II error is 0.614 (about 61.4 percent of all mergers).

Adjusting UPP for average efficiencies for both merger partners (second column, lower row in Figure 4), the probability of false positives declines to 0.175 , probability of false negatives increases to 0.092 , and total probability of type I and type II error decreases to 0.267 . This is what may be expected using the current practice of efficiency credits (in the scenario with 

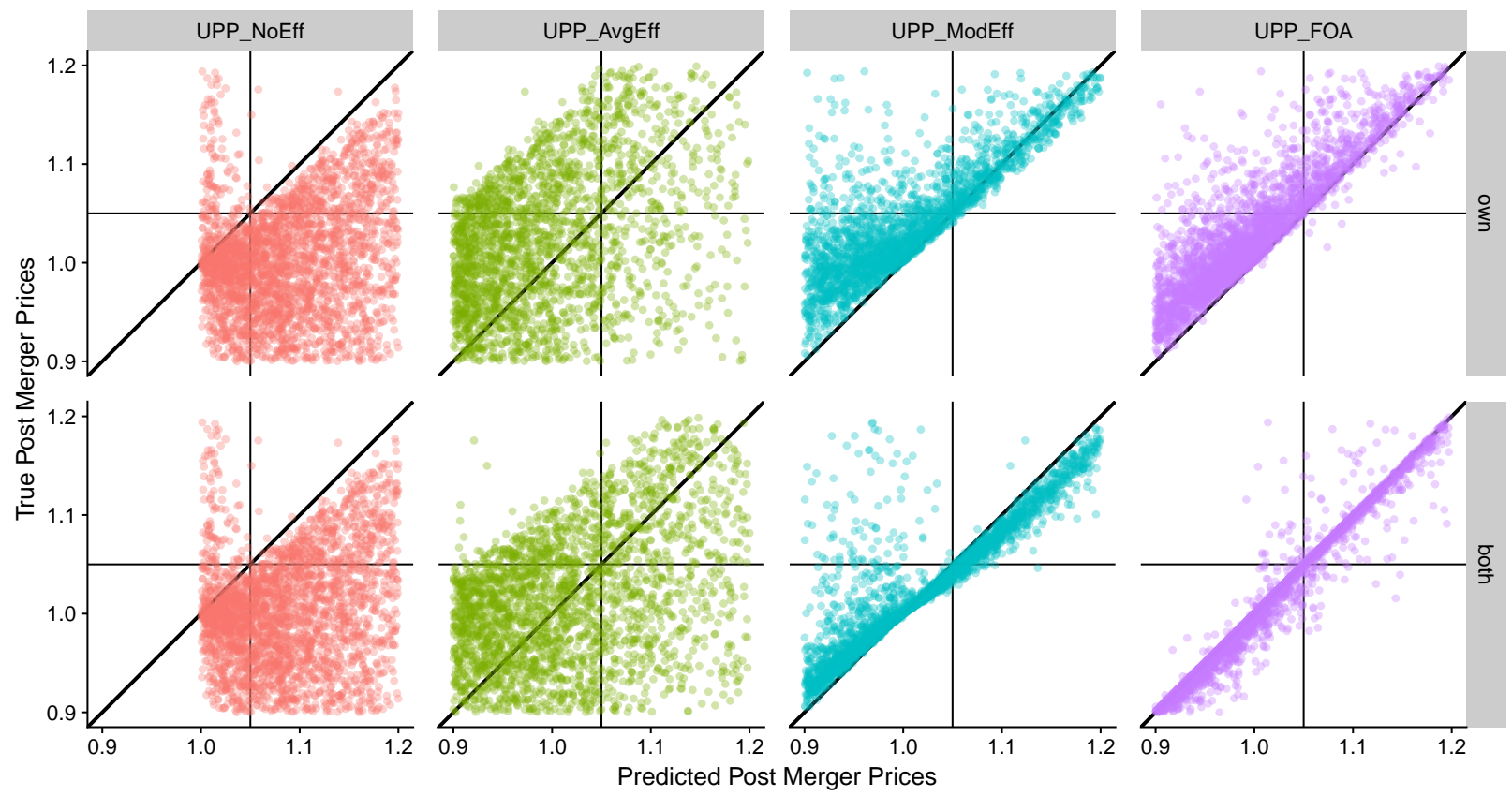

Figure 4: 5\% Threshold on Decision Rule for Price increases 
logit demand and generalized Leontief cost complementarities).

Using the UPP Modeff calculation that includes model-based cost efficiencies (third column, lower row in Figure 4), the total probability of making a type I or type II error goes down to 0.057 , and using $U P P_{F O A}$ calculation lowers this total probability even more to 0.017 (about 1.7 percent of all mergers).

In other words, total probability of making a merger screening error decreases $79 \%$ (from 0.267 to 0.057 ) as we move from current practice (using $U P P_{A v g E f f}$ ) to model-based $U P P_{M o d E f f}$ and decreases $94 \%$ (from 0.267 to 0.017 ) as we move from current practice to $U P P_{F O A}$. These results are based on logit demand and generalized Leontief costs. A similar pattern is seen for many of the other scenarios as well, as documented in Table 3. Notably, in six of the eight scenarios, using $U P P_{F O A}$ reduces total probability of false positive and false negatives to less than 0.02 (The exceptional cases are still the ones with log-linear demand as discussed above). The average reduction in total probability of making an error in these cases is $96 \%$.

Moreover, in four scenarios, using $U P P_{F O A}$ (over $U P P_{A v g E f f}$ ) reduces total probability of false positive and false negatives to less than 0.007 . The average reduction in making an merger screening error in these four cases is $98 \%$. (We find similar patterns with a $10 \%$ price increase threshold.)

Taken together, these results present more evidence of the benefit from using model-based efficiencies and a more accurate first-order approximation in UPP calculations.

\section{Comparison to UPP with higher efficiency thresholds}

We know that baseline UPP over-estimates actual post-merger prices, which in its turn impacts the validity ot UPP in both post-merger price estimation accuracy and merger screening accuracy. Based on the results shown in the previous sections, the probabilities associated with merger screening errors may be very large. Farrell \& Shapiro (2010) suggest "using a starkly simple default value for efficiencies" that could for example be of $10 \%$. This would allow, at least in principle, to postpone specific estimations of merger-specific efficiencies after an initial screen, similarly to suggestions in Warren-Boulton (1985). Agencies typically adjust for this by using a higher threshold at which baseline UPP is used to flag a merger for additional analysis.

Differently than for the Herfindahl Hirschman Index, to which thresholds are made publicly available at HMG(2010), thus far there is no official threshold for UPP. We consider a 5 percent threshold, due to its use as a benchmark for market definition in the hypothetical monopolist test, as described in 4.1.2 of HMG (2010); ${ }^{13}$ as well as its proximity to the optimal threshold for UPP of four percent estimated in Garmon (2017). Miller et al. (2017) analyze the occurence of false positives and negatives in upward pricing pressure by using a 10 percent threshold, as suggested in Farrell \& Shapiro (2010). Coate (2011) goes through the variety of cases that have been evaluated by the FTC from 1993 until mid-2010 and

\footnotetext{
${ }^{13}$ Despite of the Agencies saying that the small but significant non transitory increase in price (SSNIP) is a threshold for market definition, as does not reflect their tolerance towards price increase, it is still a good indicator of what could potentially be considered anticompetitive.
} 
Table 3: Improvement in Merger Screening Accuracy - 5\% Tolerance Threshold

\begin{tabular}{|c|c|c|c|c|c|c|c|c|c|}
\hline \multicolumn{10}{|c|}{ Generalized LeOntief } \\
\hline Logit Demand & NoEff & AvgEff & ModEff & FOA & Linear Demand & NoEff & AvgEff & ModEff & FOA \\
\hline Type I error & 0.579 & 0.175 & 0.022 & 0.010 & Type I error & 0.582 & 0.173 & 0.030 & 0.000 \\
\hline Type II error & 0.035 & 0.092 & 0.035 & 0.008 & Type II error & 0.000 & 0.051 & 0.004 & 0.000 \\
\hline Total & 0.614 & 0.267 & 0.057 & 0.017 & Total & 0.583 & 0.224 & 0.034 & 0.000 \\
\hline \multirow{2}{*}{\multicolumn{3}{|c|}{$\begin{array}{l}\text { Absolute Gain w.r.t. AvgEff } \\
\text { Relative Gain w.r.t. AvgEff ( } \%)\end{array}$}} & 0.210 & 0.250 & \multirow{2}{*}{\multicolumn{2}{|c|}{$\begin{array}{l}\text { Absolute Gain w.r.t. AvgEff } \\
\text { Relative Gain w.r.t. AvgEff (\%) }\end{array}$}} & & 0.190 & 0.224 \\
\hline & & & 78.78 & 93.53 & & & & 84.94 & 100.00 \\
\hline Log-Linear Demand & NoEff & AvgEff & ModEff & FOA & Almost Ideal Demand & NoEff & AvgEff & ModEff & FOA \\
\hline Type I error & 0.408 & 0.079 & 0.015 & 0.083 & Type I error & 0.430 & 0.084 & 0.014 & 0.003 \\
\hline Type II error & 0.014 & 0.157 & 0.137 & 0.042 & Type II error & 0.008 & 0.131 & 0.091 & 0.010 \\
\hline Total & 0.422 & 0.235 & 0.152 & 0.125 & Total & 0.438 & 0.215 & 0.105 & 0.013 \\
\hline \multirow{2}{*}{\multicolumn{2}{|c|}{$\begin{array}{l}\text { Absolute Gain w.r.t. AvgEff } \\
\text { Relative Gain w.r.t. AvgEff (\%) }\end{array}$}} & & 0.083 & 0.110 & \multirow{2}{*}{\multicolumn{2}{|c|}{$\begin{array}{l}\text { Absolute Gain w.r.t. AvgEff } \\
\text { Relative Gain w.r.t. AvgEff (\%) }\end{array}$}} & & 0.110 & 0.202 \\
\hline & & & 35.41 & 46.91 & & & & 51.30 & 93.90 \\
\hline \multicolumn{10}{|c|}{ QUADRATIC } \\
\hline Logit Demand & NoEff & AvgEff & ModEff & FOA & Linear Demand & NoEff & AvgEff & ModEff & FOA \\
\hline Type I error & 0.334 & 0.172 & 0.075 & 0.006 & $\overline{\text { Typ }}$ & 0.402 & 0.238 & 0.143 & 0.000 \\
\hline Type II error & 0.000 & 0.019 & 0.000 & 0.001 & Type II error & 0.000 & 0.017 & 0.000 & 0.000 \\
\hline Total & 0.334 & 0.190 & 0.075 & 0.007 & Total & 0.402 & 0.255 & 0.143 & 0.000 \\
\hline \multirow{2}{*}{\multicolumn{2}{|c|}{$\begin{array}{l}\text { Absolute Gain w.r.t. AvgEff } \\
\text { Relative Gain w.r.t. AvgEff (\%) }\end{array}$}} & & 0.115 & 0.184 & \multirow{2}{*}{\multicolumn{2}{|c|}{$\begin{array}{l}\text { Absolute Gain w.r.t. AvgEff } \\
\text { Relative Gain w.r.t. AvgEff (\%) }\end{array}$}} & & 0.112 & 0.255 \\
\hline & & & 60.61 & 96.53 & & & & 43.80 & 100.00 \\
\hline Log-Linear Demand & NoEff & AvgEff & ModEff & $\mathrm{FOA}$ & Almost Ideal Demand & NoEff & AvgEff & ModEff & FOA \\
\hline Type I error & 0.062 & 0.034 & 0.031 & 0.015 & Type I error & 0.122 & 0.041 & 0.031 & 0.003 \\
\hline Type II error & 0.052 & 0.208 & 0.281 & 0.112 & Type II error & 0.010 & 0.114 & 0.176 & 0.003 \\
\hline Total & 0.114 & 0.242 & 0.311 & 0.126 & Total & 0.132 & 0.155 & 0.206 & 0.007 \\
\hline \multirow{2}{*}{\multicolumn{2}{|c|}{$\begin{array}{l}\text { Absolute Gain w.r.t. AvgEff } \\
\text { Relative Gain w.r.t. AvgEff (\%) }\end{array}$}} & & -0.069 & 0.116 & \multirow{2}{*}{\multicolumn{2}{|c|}{$\begin{array}{l}\text { Absolute Gain w.r.t. AvgEff } \\
\text { Relative Gain w.r.t. AvgEff (\%) }\end{array}$}} & & -0.051 & 0.148 \\
\hline & & & -28.48 & 47.87 & & & & -33.12 & 95.75 \\
\hline
\end{tabular}


Table 4: Baseline UPP with Higher Thresholds

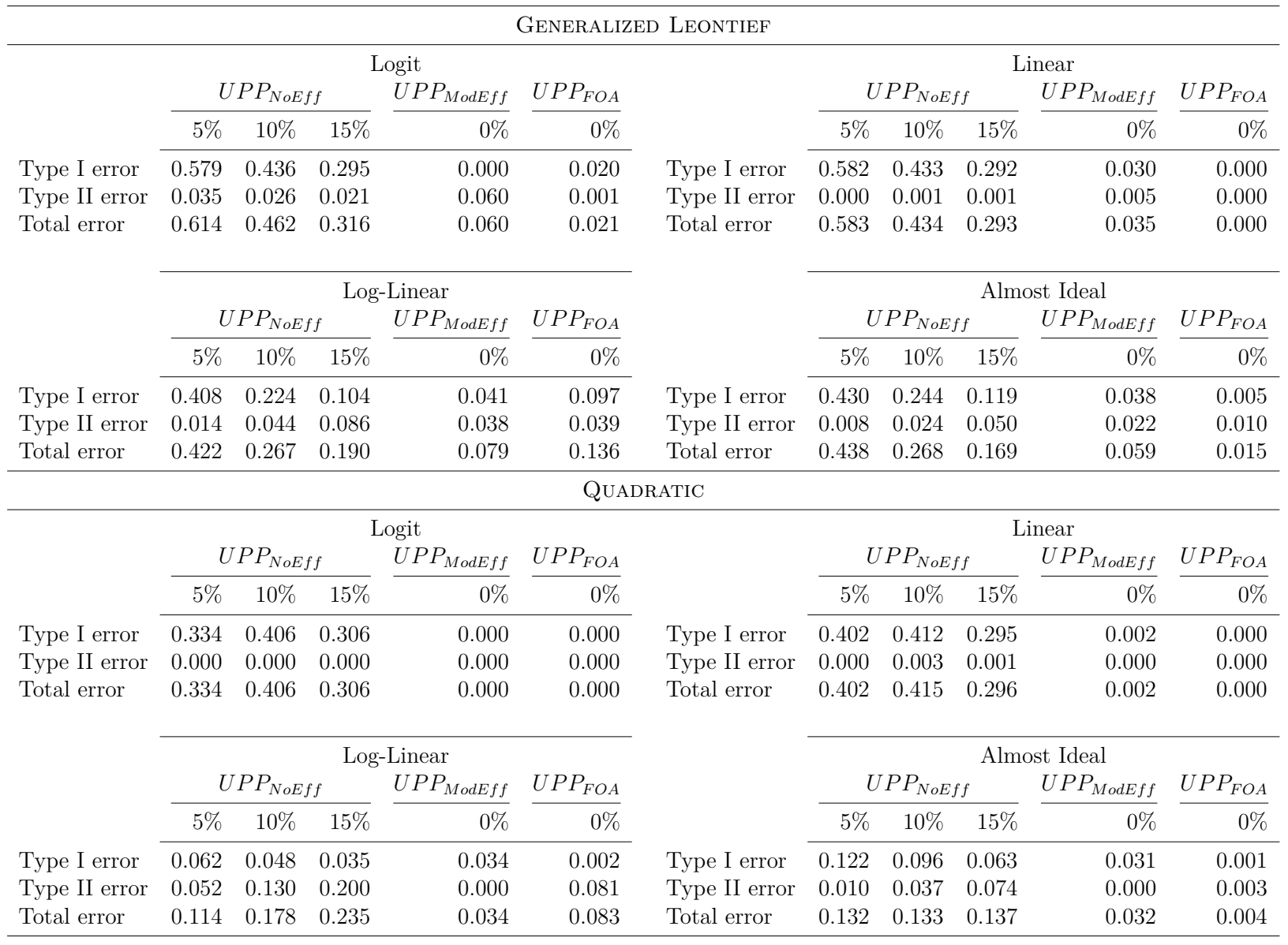

Table shows the percentage of error types in merger screening for UPP baseline formulation (for 5, 10, and $15 \%$ tolerance threshold) when compared to UPP with model-based efficiencies and first-order approximation (with a strict tolerance of $0 \%$ threshold).

attempts to determine what seems to be the implicit benchmark used for UPP; and generate a benchmark of 15 percent from the merger challenge data.

In order to provide a comparison to the current practice of using $U P P_{N o E f f}$ but including a higher price increase threshold to adjust for cost efficiencies, we compare probability of type I and II errors using price increase thresholds of 5\%,10\%, and $15 \%$ for $U P P_{N o E f f}$ with a $0 \%$ threshold for $U P P_{\text {Modeff }}$ and $U P P_{F O A}$. As shown in Table 4, in each of the eight scenarios, total probability of making type I and II errors with a $0 \%$ threshold for $U P P_{M o d E f f}$ and $U P P_{F O A}$ is lower than with a $15 \%$ threshold for $U P P_{N o E f f}$, and in many cases it is substantially lower. 


\section{Conclusion}

We investigate the accuracy of UPP as a tool in antitrust analysis by extending the standard UPP formulation to include model-based, merger-specific cost efficiencies. Using the theoretical framework in Jaffe \& Weyl (2013), we include cost efficiencies in a tractable manner and derive the related UPP formulations.

The efficacy of the new UPP formulations is analyzed using Monte Carlo simulation for 8 different scenarios; four demand systems (Logit, Linear, Log-Linear, and Almost ideal) and two merger-specific, cost complementarity systems (Generalized Leontief and Quadratic). For each scenario we simulate 5,000 mergers, for a total of 40,000 mergers.

We find that the new UPP formulations yield substantial gains in post-merger price prediction, as compared to existing practice, and yield substantial gains in merger screening accuracy as well. Moreover, the new UPP formulations outperform the standard UPP formulation at higher thresholds for the cases considered here. The results support the inclusion

of model-based cost efficiencies in the standard UPP formulation for more accurate antitrust decision-making. 


\section{References}

Berry, S. \& Pakes, A. (1993). Some applications and limitations of recent advances in empirical industrial organization: Merger analysis. The American Economic Review, 83(2), $247-252$.

Buccirossi, P. (2008). Handbook of antitrust economics.

Budzinski, O. \& Ruhmer, I. (2009). Merger simulation in competition policy: A survey. Journal of Competition Law and Economics, 6(2), 277-319.

Caves, D. W., Christensen, L. R., \& Tretheway, M. W. (1980). Flexible cost functions for multiproduct firms. The Review of Economics and Statistics, (pp. 477-481).

Cheung, L. (2016). An empirical comparison between the upward pricing pressure test and merger simulation in differentiated product markets. Journal of Competition Law $\mathcal{E}$ Economics, 12(4), 701-734.

Coate, M. B. (2011). Benchmarking the upward pricing pressure model with federal trade commission evidence. Journal of Competition Law and economics, 7(4), 825-846.

Cohn, E., Rhine, S. L., \& Santos, M. C. (1989). Institutions of higher education as multiproduct firms: Economies of scale and scope. The review of economics and statistics, (pp. 284-290).

Crane, D. A. (2011). Rethinking merger efficiencies. Michigan Law Review, (pp. 347-391).

Deneckere, R. \& Davidson, C. (1985). Incentives to form coalitions with bertrand competition. The RAND Journal of economics, (pp. 473-486).

Diewert, W. E. (1971). An application of the shephard duality theorem: A generalized leontief production function. Journal of political Economy, 79(3), 481-507.

Epstein, R. J. \& Rubinfeld, D. L. (2001). Merger simulation: A simplified approach with new applications. Antitrust LJ, 69, 883.

Farrell, J. \& Shapiro, C. (2001). Scale economies and synergies in horizontal merger analysis. Antitrust Law Journal, 68(3), 685-710.

Farrell, J. \& Shapiro, C. (2010). Antitrust evaluation of horizontal mergers: An economic alternative to market definition. The BE Journal of Theoretical Economics, 10(1).

Farrell, J., Shapiro, C., et al. (2011). Upward pricing pressure and critical loss analysis: Response. Antitrust Chronicle, 1.

Garmon, C. (2017). The accuracy of hospital merger screening methods. The RAND Journal of Economics, 48(4), 1068-1102.

Gifford, D. J. \& Kudrle, R. T. (2015). The Atlantic Divide in Antitrust: An Examination of US and EU Competition Policy. University of Chicago Press. 
Hall, R. E. (1973). The specification of technology with several kinds of output. Journal of Political Economy, 81(4), 878-892.

Hausman, J., Leonard, G., \& Zona, J. D. (1994). Competitive analysis with differenciated products. Annales d'Economie et de Statistique, (pp. 159-180).

Hausman, J. A. \& Leonard, G. K. (1996). Economic analysis of differentiated products mergers using real world data. Geo. Mason L. Rev., 5, 321.

Ivaldi, M., Jullien, B., Rey, P., Seabright, P., Tirole, J., et al. (2003). The economics of unilateral effects. Interim report for DG competition, European Commission.

Jaffe, S. \& Weyl, E. G. (2013). The first-order approach to merger analysis. American Economic Journal: Microeconomics, 5(4), 188-218.

Kinne, K. (1999). Efficiencies in merger analysis. Intereconomics, 34(6), 297-302.

Li, T. \& Rosenman, R. (2001). Estimating hospital costs with a generalized leontief function. Health Economics, 10(6), 523-538.

Martínez-Budría, E., Jara-Díaz, S., \& Ramos-Real, F. J. (2003). Adapting productivity theory to the quadratic cost function. an application to the spanish electric sector. Journal of Productivity Analysis, 20(2), 213-229.

Miller, N. H., Remer, M., Ryan, C., \& Sheu, G. (2013a). On the first order approximation of counterfactual price effects in oligopoly models.

Miller, N. H., Remer, M., Ryan, C., \& Sheu, G. (2016). Pass-through and the prediction of merger price effects. The Journal of Industrial Economics, 64(4), 683-709.

Miller, N. H., Remer, M., Ryan, C., \& Sheu, G. (2017). Upward pricing pressure as a predictor of merger price effects. International Journal of Industrial Organization, 52, $216-247$.

Miller, N. H., Remer, M., \& Sheu, G. (2013b). Using cost pass-through to calibrate demand. Economics Letters, 118(3), 451-454.

Moresi, S. \& Salop, S. C. (2013). vguppi: Scoring unilateral pricing incentives in vertical mergers. Antitrust LJ, 79, 185.

Nevo, A. (2000). Mergers with differentiated products: The case of the ready-to-eat cereal industry. The RAND Journal of Economics, (pp. 395-421).

Schmalensee, R. et al. (2009). Should new merger guidelines give upp market definition? CPI Antitrust Chronicle, 12(1).

Shapiro, C. (2010). The 2010 horizontal merger guidelines: From hedgehog to fox in forty years. Antitrust Law Journal, 77(1), 49-107. 
Simons, J. J. \& Coate, M. B. (2010). Upward pressure on price analysis: issues and implications for merger policy. European Competition Journal, 6(2), 377-396.

Warren-Boulton, F. R. (1985). Merger policy and enforcement at the antitrust division: The economist's view. Antitrust LJ, 54, 109.

Weinberg, M. C. \& Hosken, D. (2013). Evidence on the accuracy of merger simulations. Review of Economics and Statistics, 95(5), 1584-1600.

Werden, G. J. (1996). A robust test for consumer welfare enhancing mergers among sellers of differentiated products. The Journal of Industrial Economics, (pp. 409-413).

Werden, G. J. (2010). Unilateral competitive effects of horizontal mergers i: Basic concepts and models.

Werden, G. J. \& Froeb, L. M. (1994). The effects of mergers in differentiated products industries: Logit demand and merger policy. Journal of Law, Economics, ES Organization, (pp. 407-426).

Werden, G. J. \& Froeb, L. M. (1996). Simulation as an alternative to structural merger policy in differentiated products industries. In The Economics of the Antitrust Process (pp. 65-88). Springer.

Werden, G. J., Froeb, L. M., \& Scheffman, D. T. (2003). A daubert discipline for merger simulation. Antitrust, 18, 89.

Willig, R. (2011). Unilateral competitive effects of mergers: Upward pricing pressure, product quality, and other extensions. Review of Industrial Organization, 39(1-2), 19. 


\section{A Appendix}
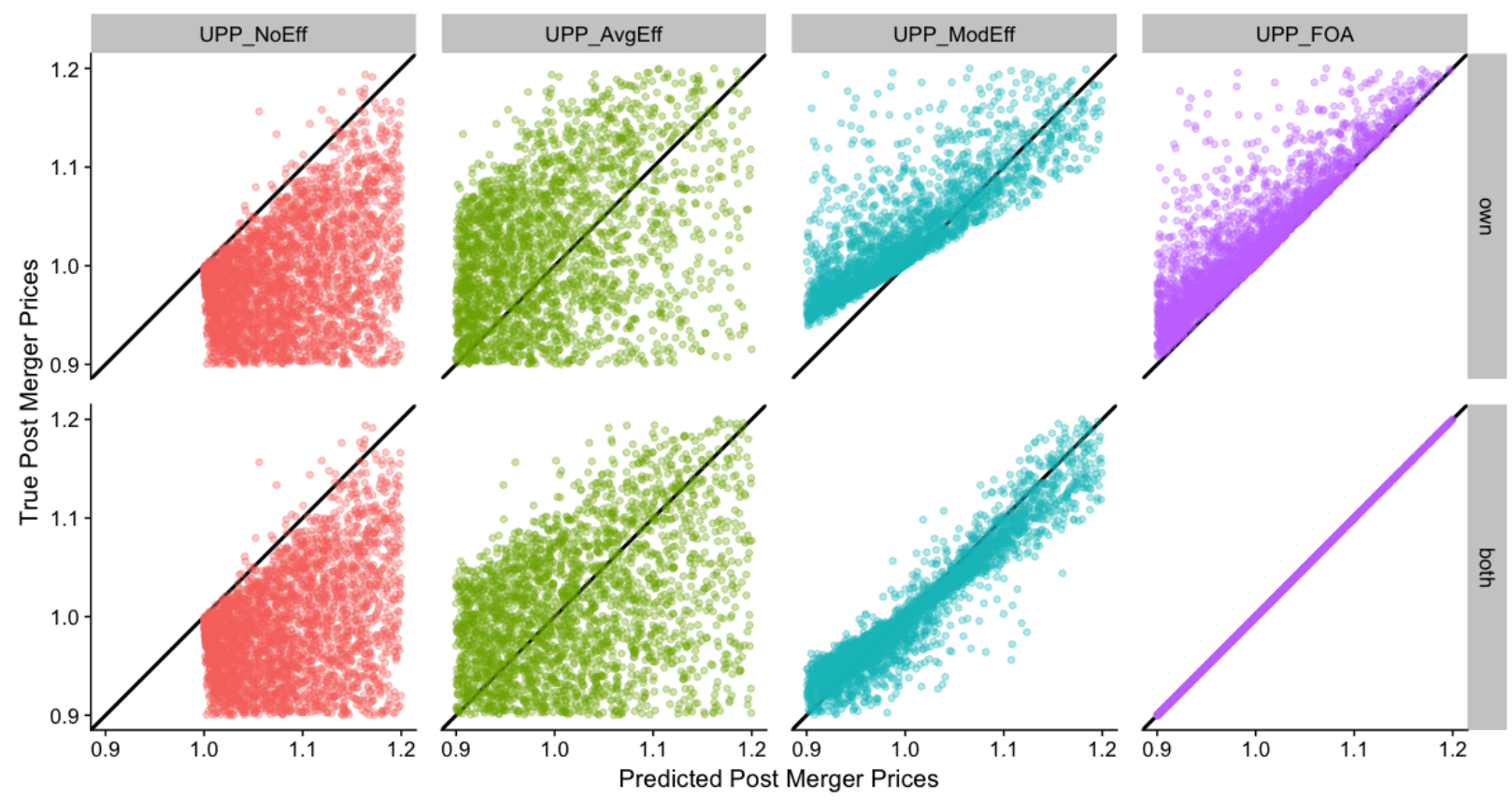

Figure 5: Accuracy of Prediction - Linear Demand, Generalized Leontief Cost

First row shows the distribution of the true post merger prices against the predicted post merger prices using different UPP calculations and own goods' efficiencies included in the computation. Second row shows the same for both goods' efficiencies. 


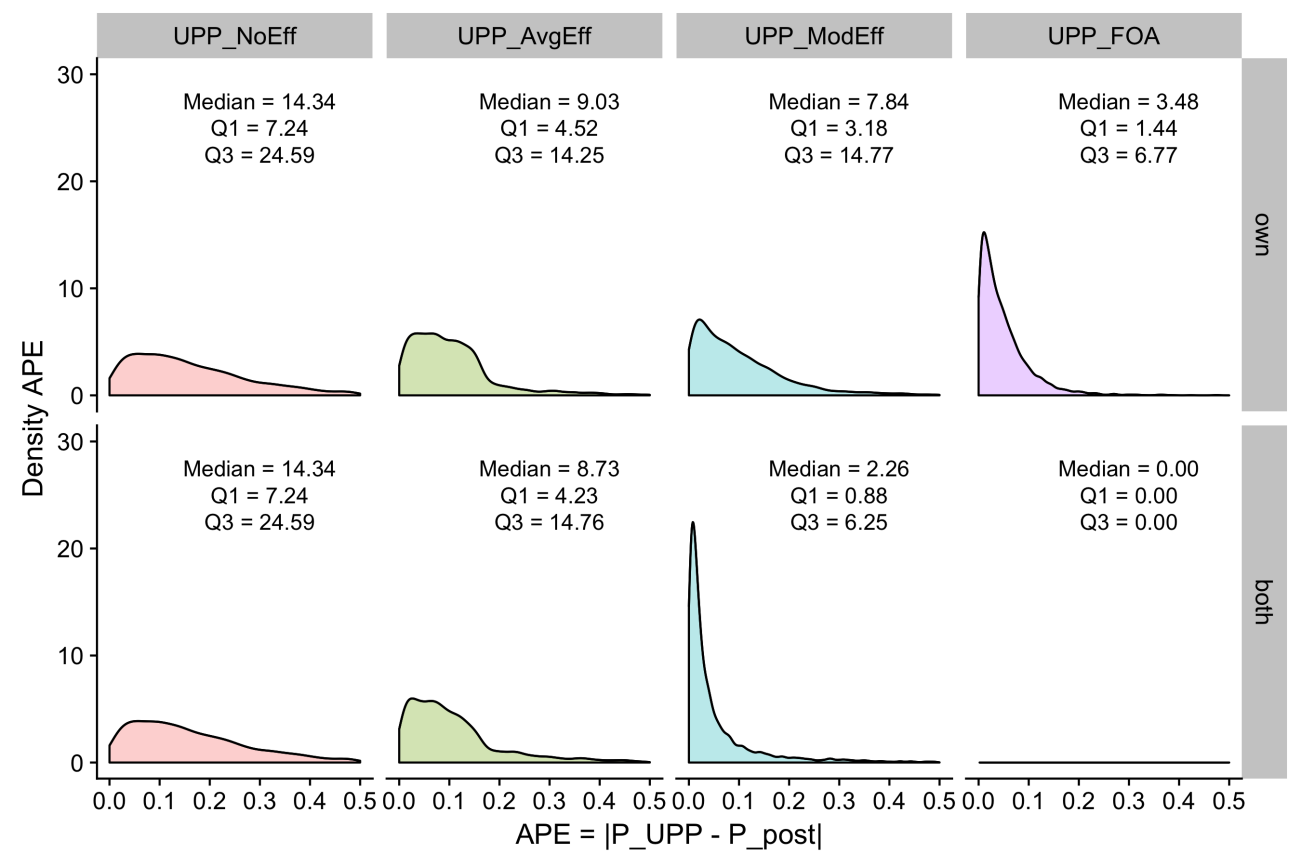

Figure 6: Absolute Prediction Errors - Linear Demand, Generalized Leontief Cost

Portrays density kernels for absolute prediction errors, as well as the median absolute prediction error, first and third quartile for each specification.

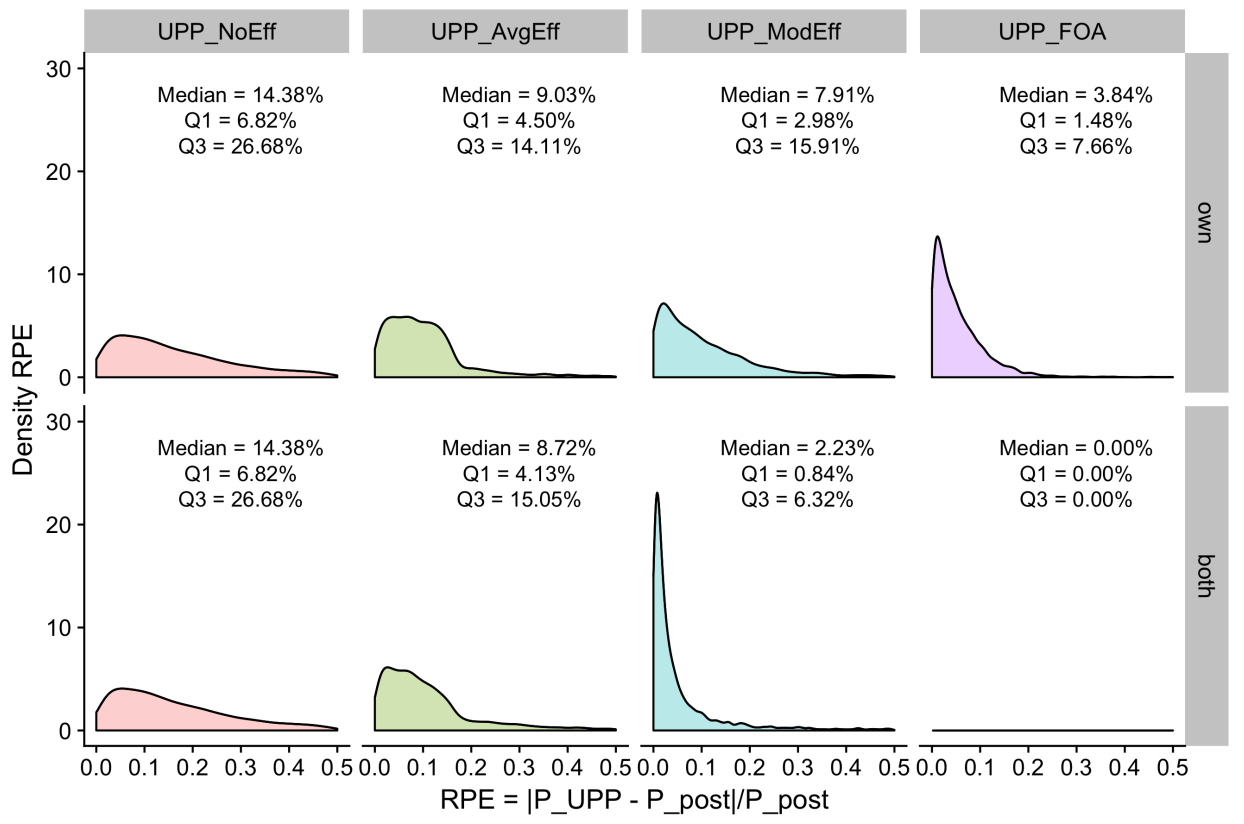

Figure 7: Relative Prediction Errors - Linear Demand, Generalized Leontief Cost

Portrays density kernels for relative prediction errors, as well as the median relative prediction error, first and third quartile for each specification. 

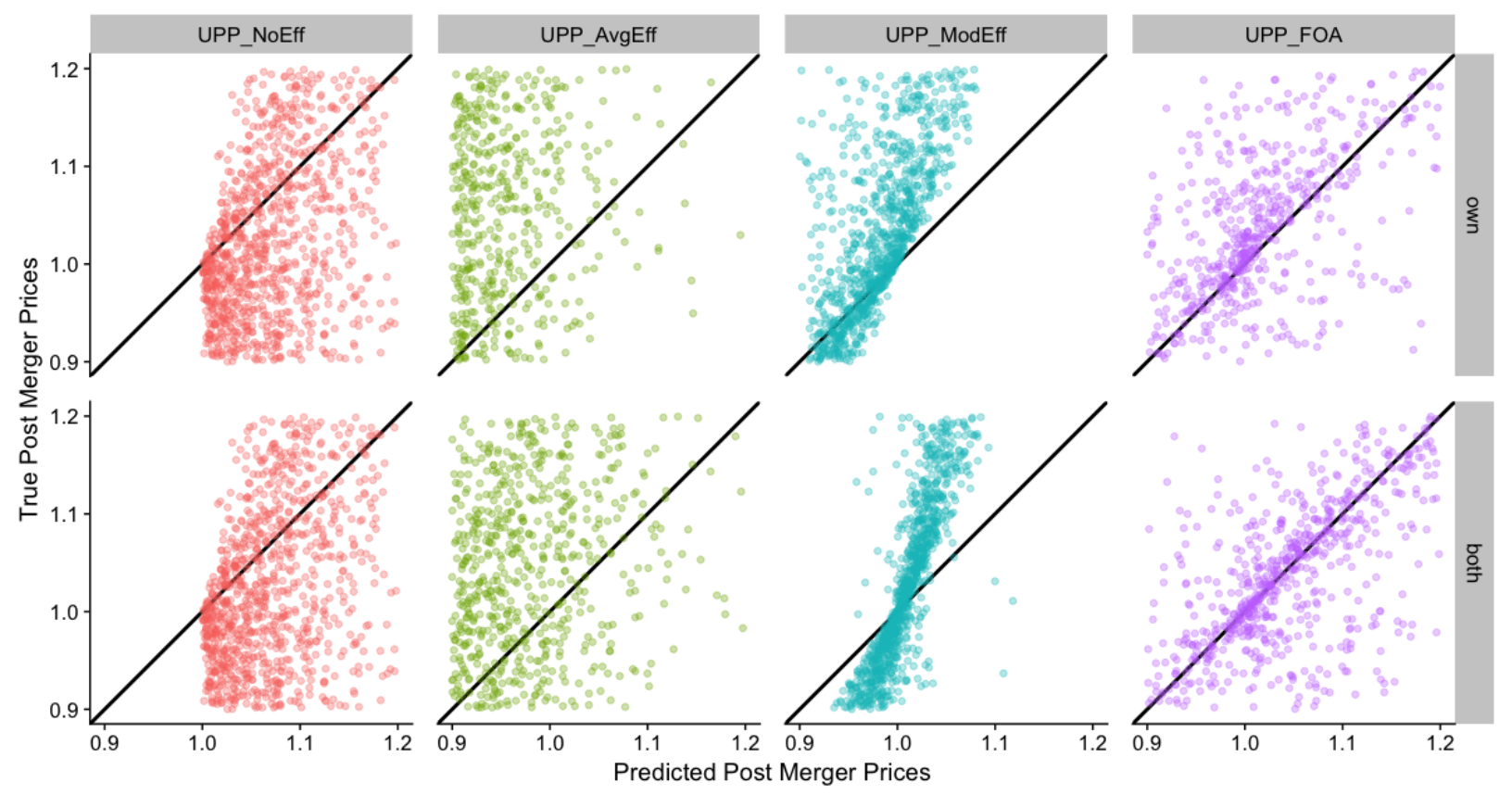

Figure 8: Accuracy of Prediction - Log-Linear Demand, Generalized Leontief Cost

First row shows the distribution of the true post merger prices against the predicted post merger prices using different UPP calculations and own goods' efficiencies included in the computation. Second row shows the same for both goods' efficiencies. 


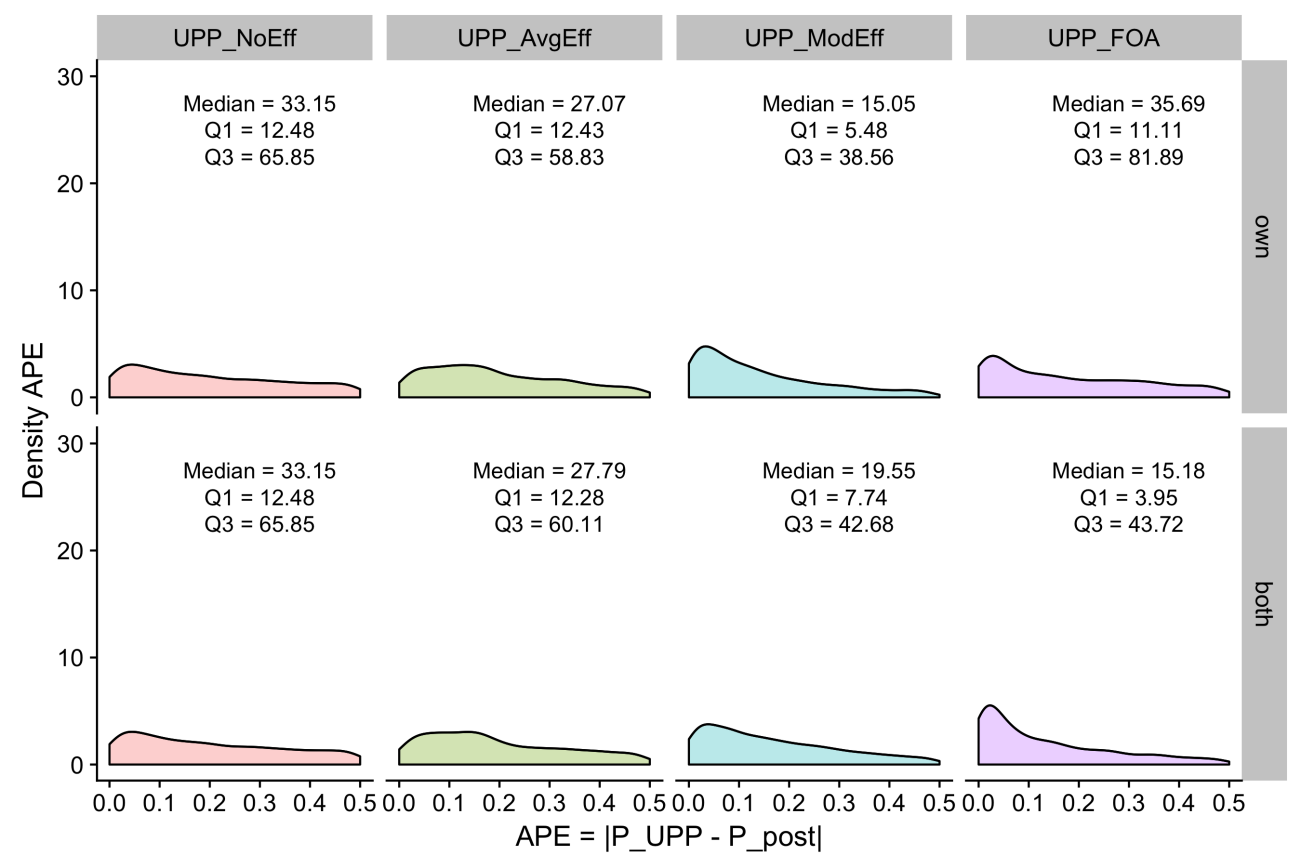

Figure 9: Absolute Prediction Errors - Log-Linear Demand, Generalized Leontief Cost

Portrays density kernels for absolute prediction errors, as well as the median absolute prediction error, first and third quartile for each specification.

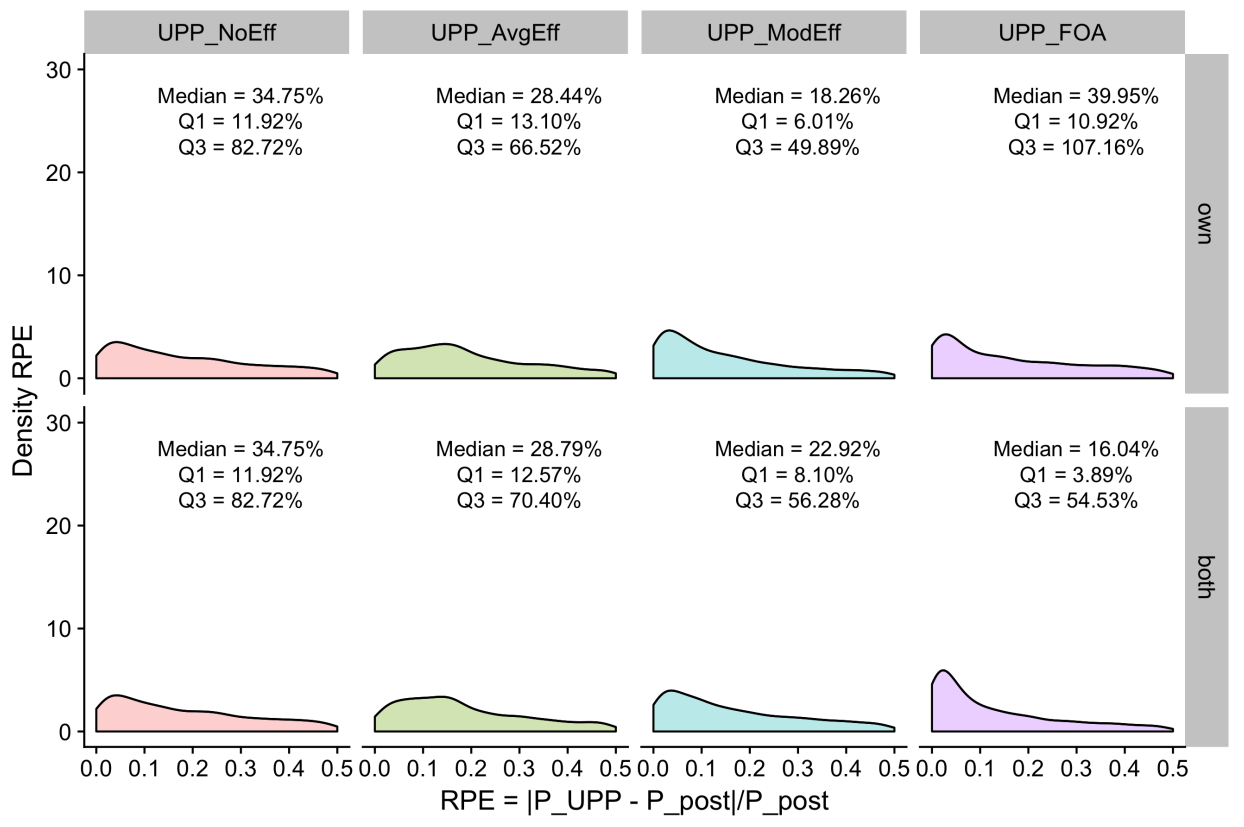

Figure 10: Relative Prediction Errors - Log-Linear Demand, Generalized Leontief Cost

Portrays density kernels for relative prediction errors, as well as the median, relative prediction error, first and third quartile for each specification. 

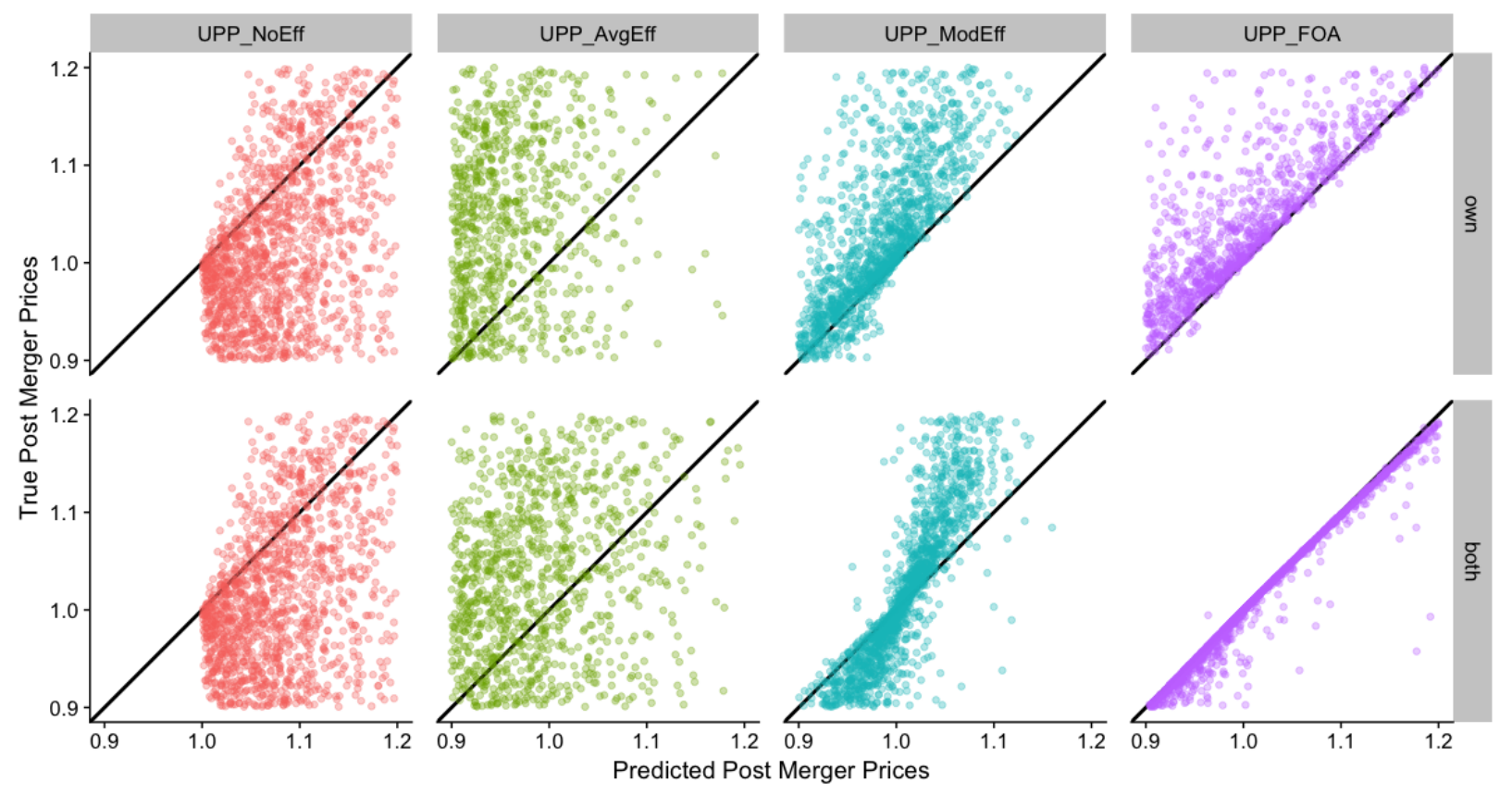

Figure 11: Accuracy of Prediction - Almost Ideal Demand, Generalized Leontief Cost

First row shows the distribution of the true post merger prices against the predicted post merger prices using different UPP calculations and own goods' efficiencies included in the computation. Second row shows the same for both goods' efficiencies. 


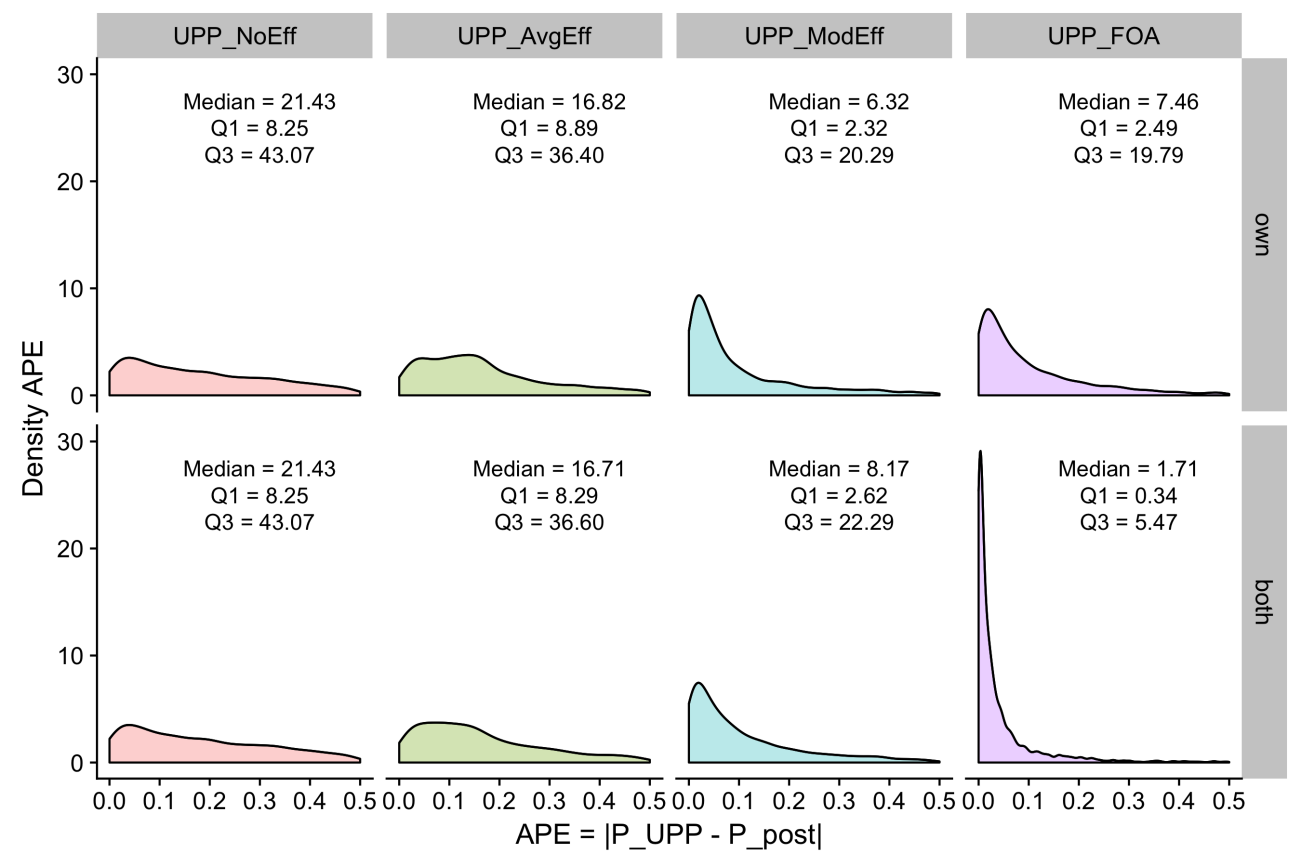

Figure 12: Absolute Prediction Errors - Log-Linear Demand, Generalized Leontief Cost

Portrays density kernels for absolute prediction errors, as well as the median absolute prediction error, first and third quartile for each specification.

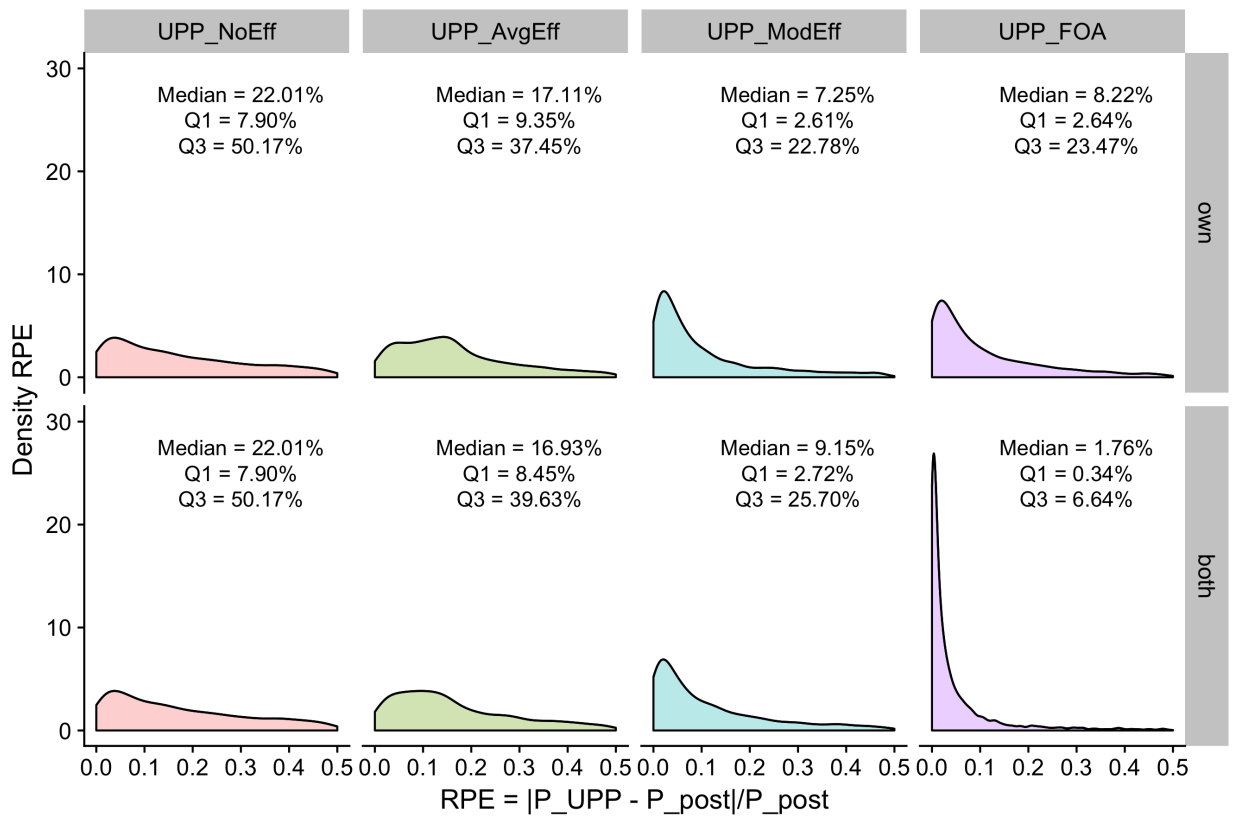

Figure 13: Relative Prediction Errors - Log-Linear Demand, Generalized Leontief Cost

Portrays density kernels for relative prediction errors, as well as the median relative prediction error, first and third quartile for each specification. 

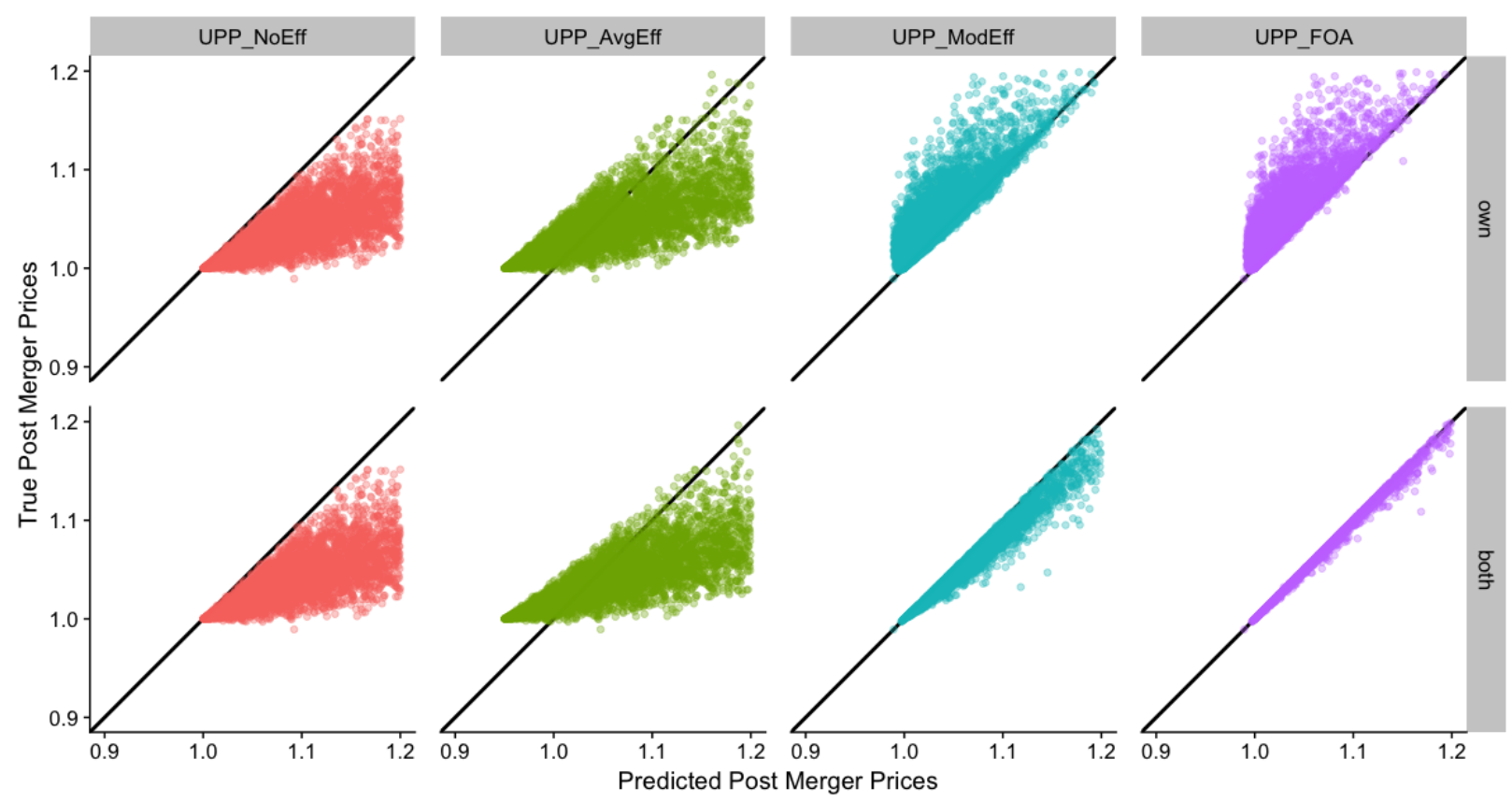

Figure 14: Accuracy of Prediction - Logit Demand, Quadratic Cost

First row shows the distribution of the true post merger prices against the predicted post merger prices using different UPP calculations and own goods' efficiencies included in the computation. Second row shows the same for both goods' efficiencies. 


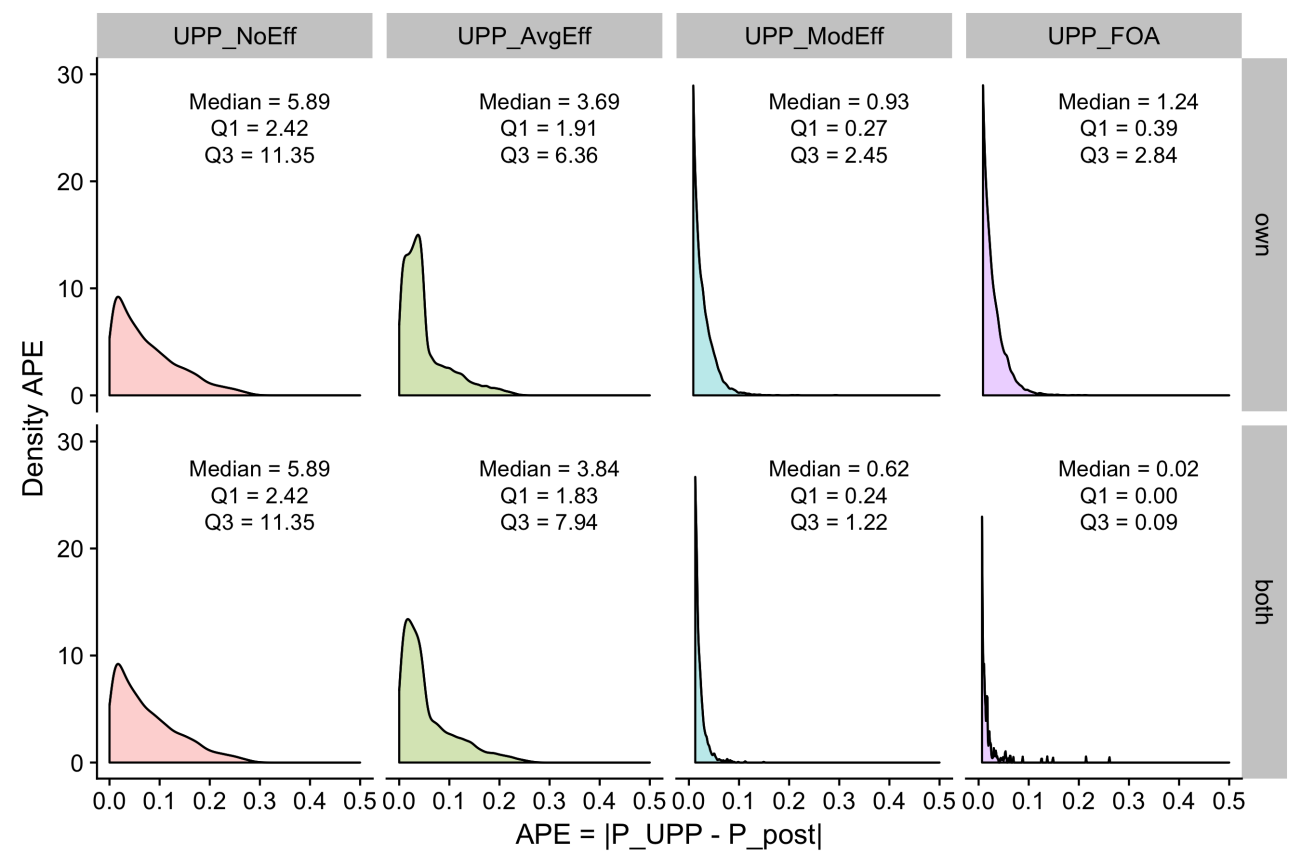

Figure 15: Absolute Prediction Errors - Logit Demand, Quadratic Cost

Portrays density kernels for absolute prediction errors, as well as the median absolute prediction error, first and third quartile for each specification.

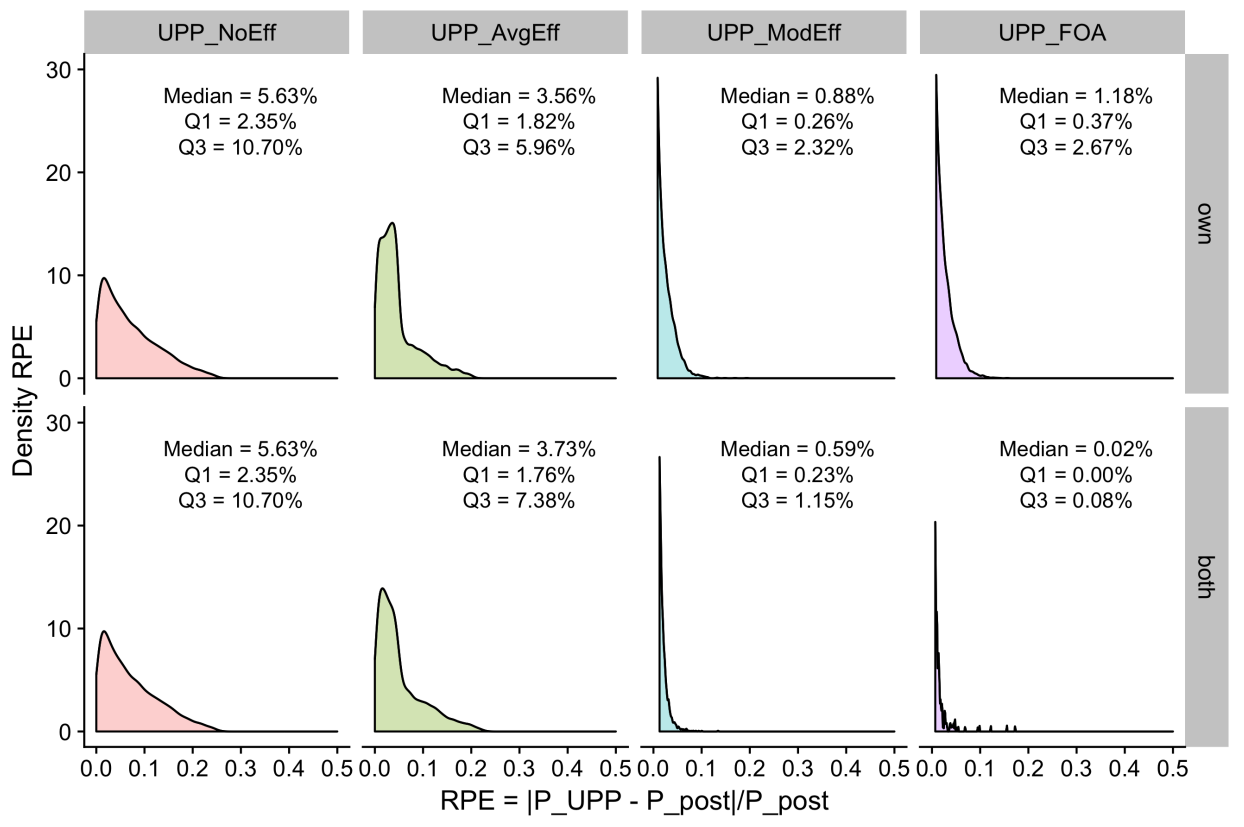

Figure 16: Relative Prediction Errors - Logit Demand, Quadratic Cost

Portrays density kernels for relative prediction errors, as well as the median relative prediction error, first and third quartile for each specification. 

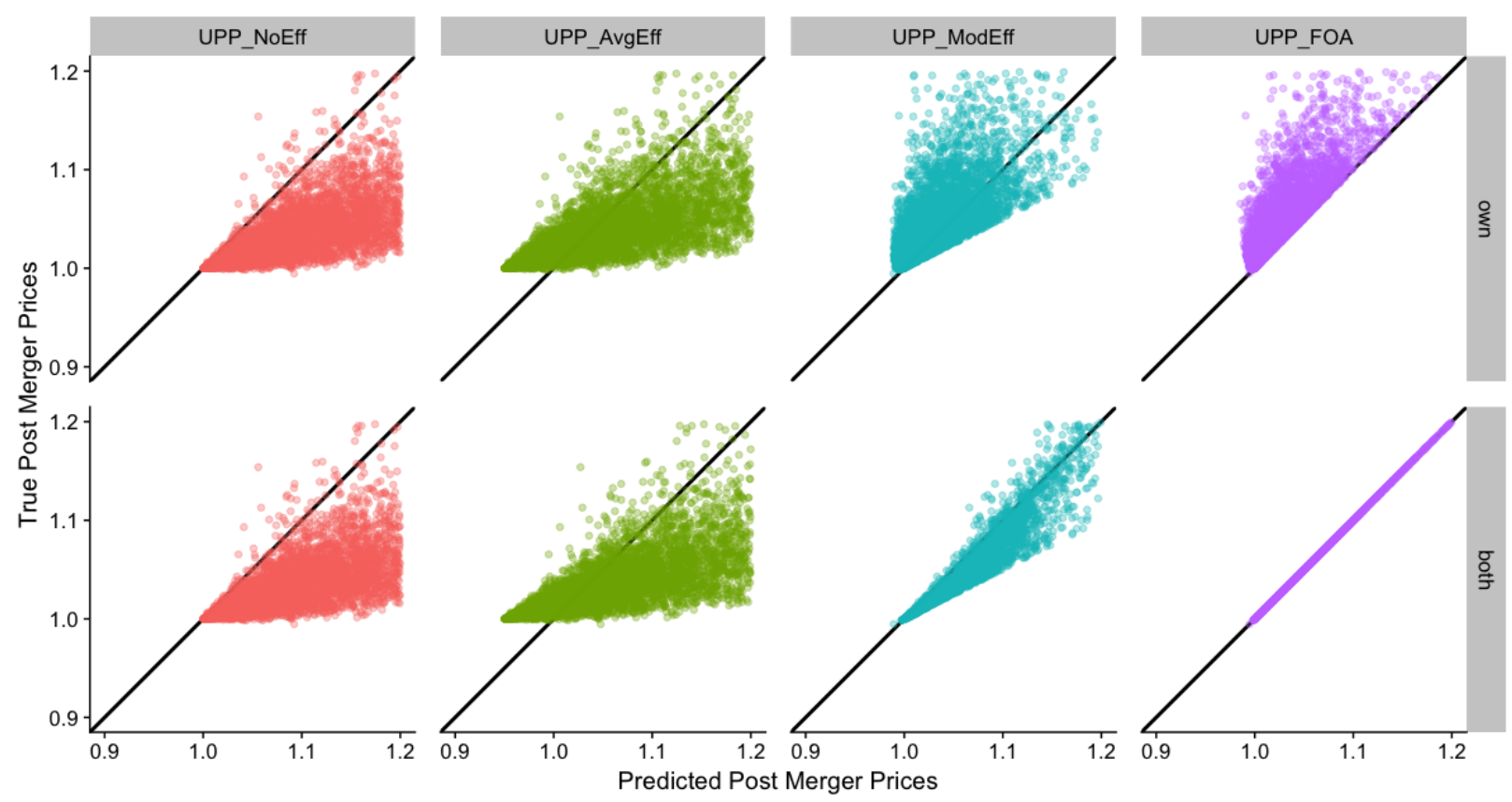

Figure 17: Accuracy of Prediction - Linear Demand, Quadratic Cost

First row shows the distribution of the true post merger prices against the predicted post merger prices using different UPP calculations and own goods' efficiencies included in the computation. Second row shows the same for both goods' efficiencies. 


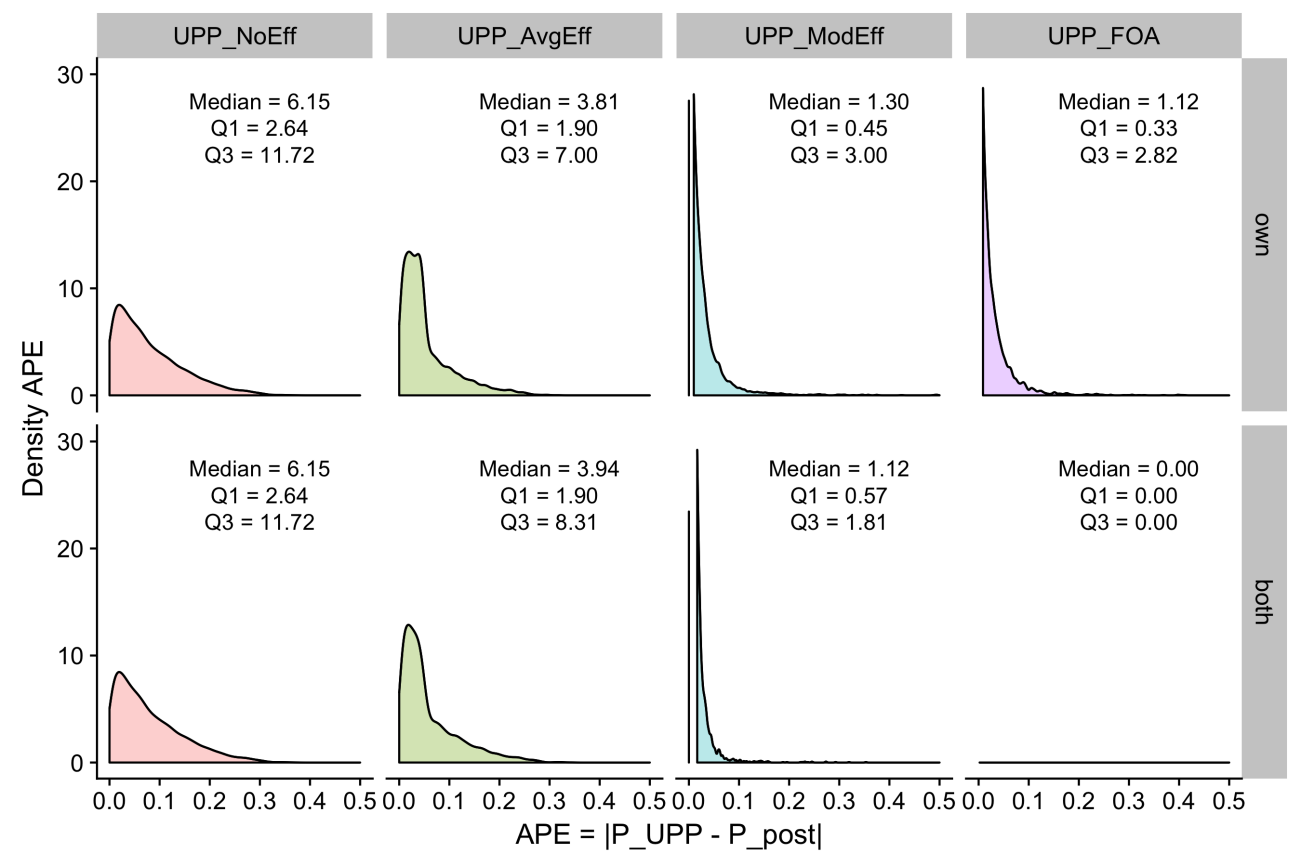

Figure 18: Absolute Prediction Errors - Linear Demand, Quadratic Cost

Portrays density kernels for absolute prediction errors, as well as the median absolute prediction error, first and third quartile for each specification.

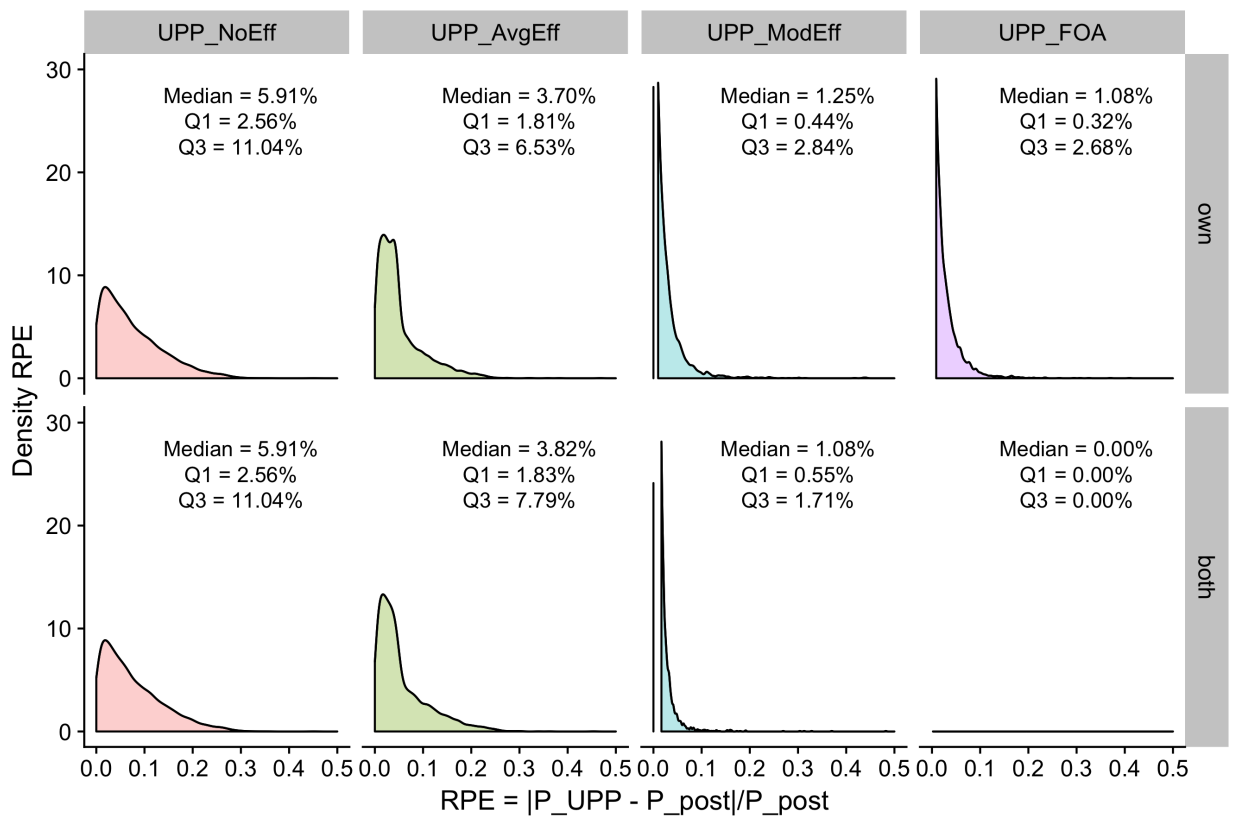

Figure 19: Relative Prediction Errors - Linear Demand, Quadratic Cost

Portrays density kernels for relative prediction errors, as well as the median relative prediction error, first and third quartile for each specification. 

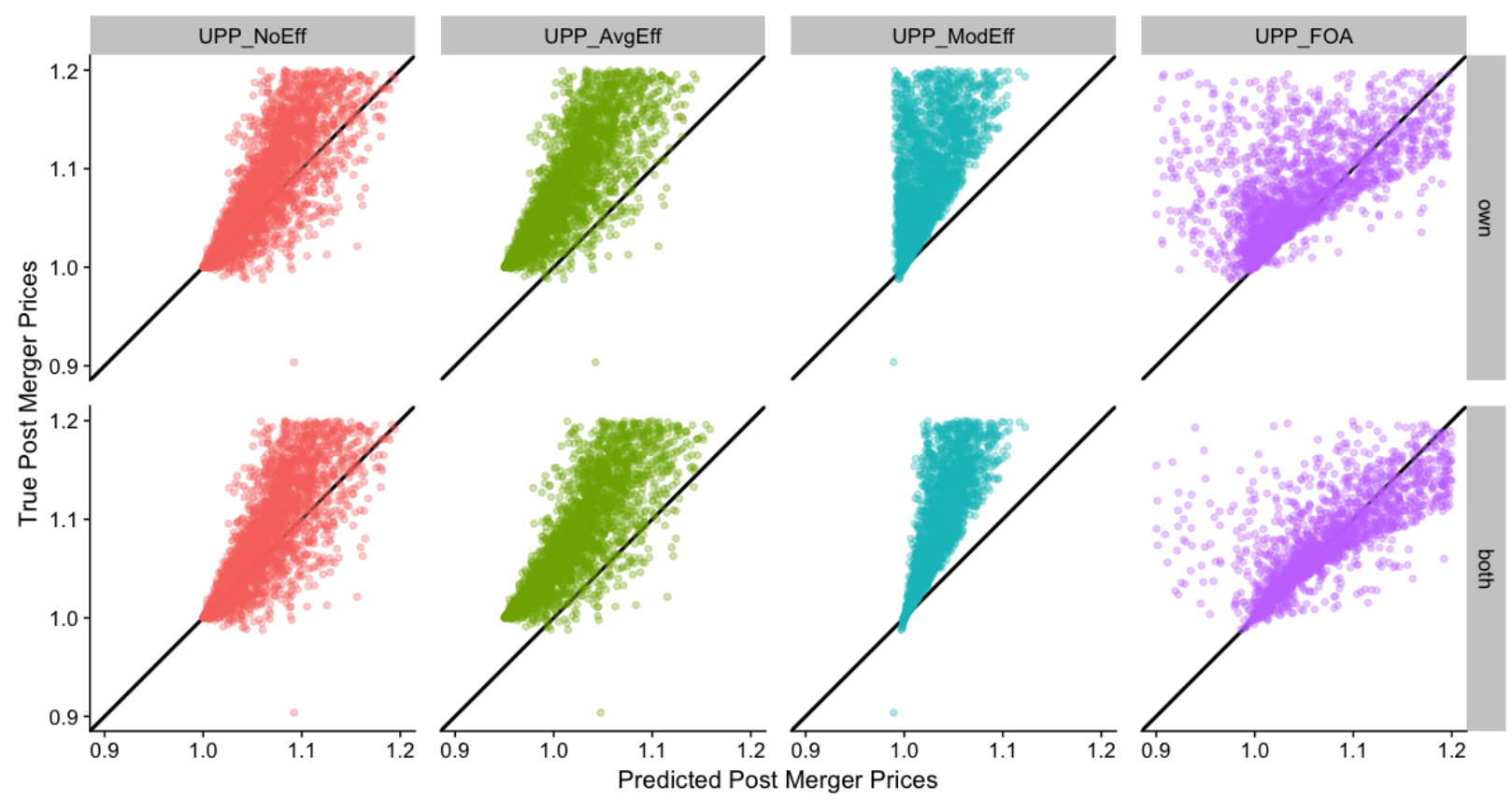

Figure 20: Accuracy of Prediction - Log-Linear Demand, Quadratic Cost

First row shows the distribution of the true post merger prices against the predicted post merger prices using different UPP calculations and own goods' efficiencies included in the computation. Second row shows the same for both goods' efficiencies. 


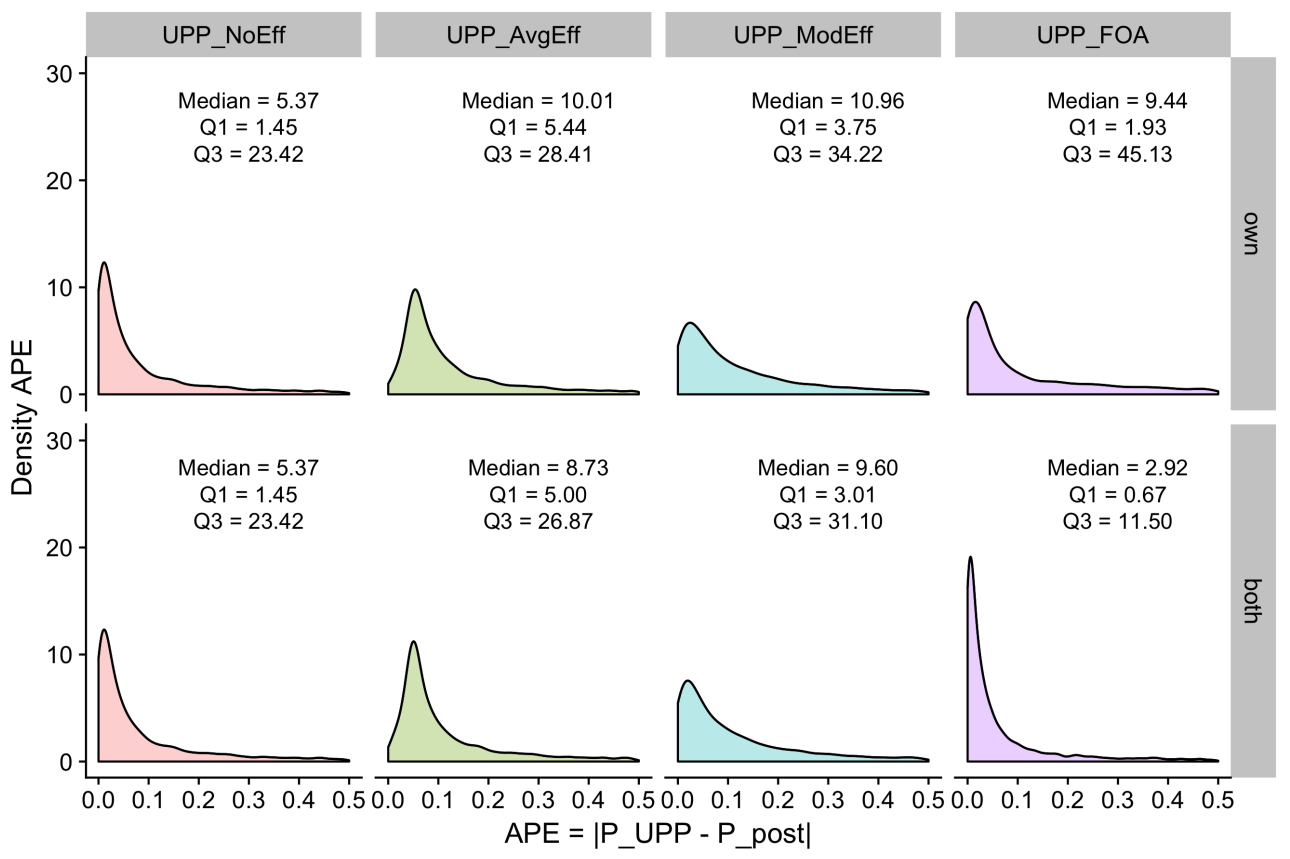

Figure 21: Absolute Prediction Errors - Log-Linear Demand, Quadratic Cost

Portrays density kernels for absolute prediction errors, as well as the median absolute prediction error, first and third quartile for each specification.

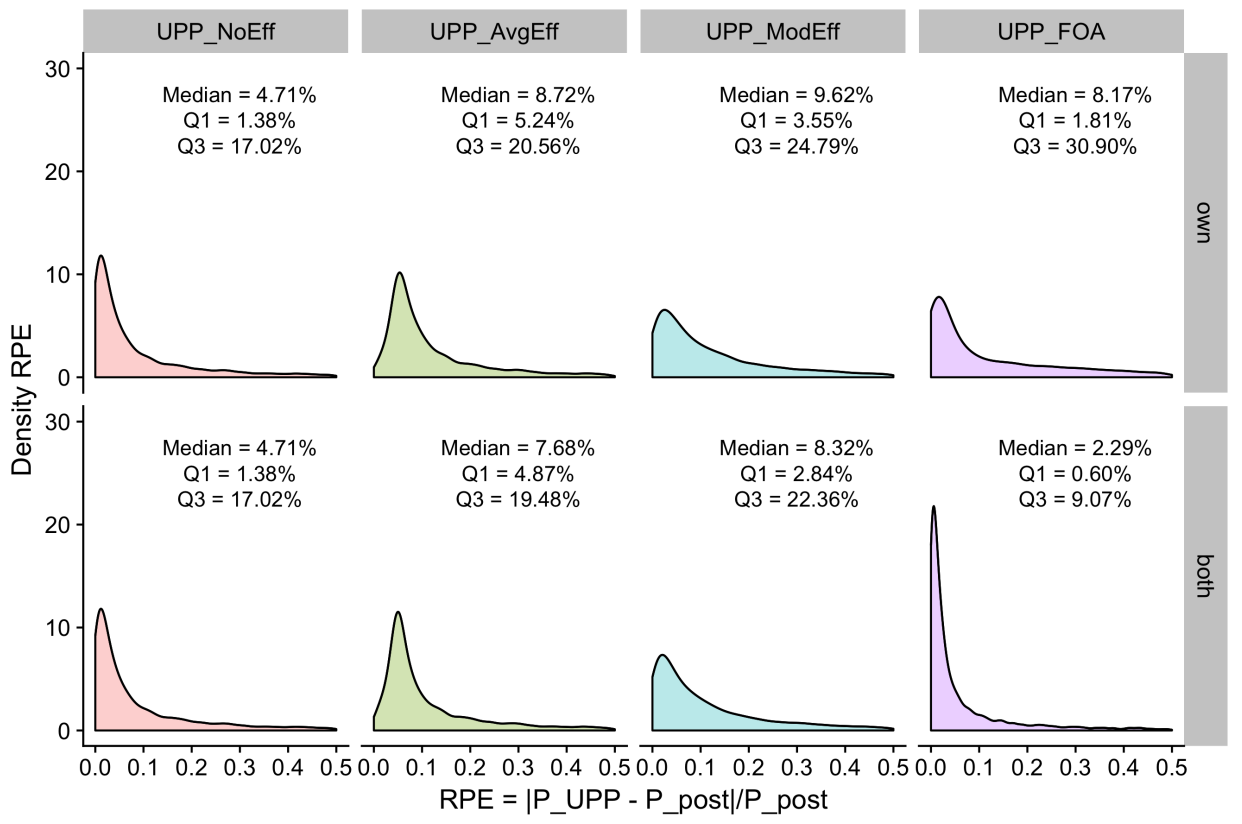

Figure 22: Relative Prediction Errors - Log-Linear Demand, Quadratic Cost

Portrays density kernels for relative prediction errors, as well as the median relative prediction error, first and third quartile for each specification. 

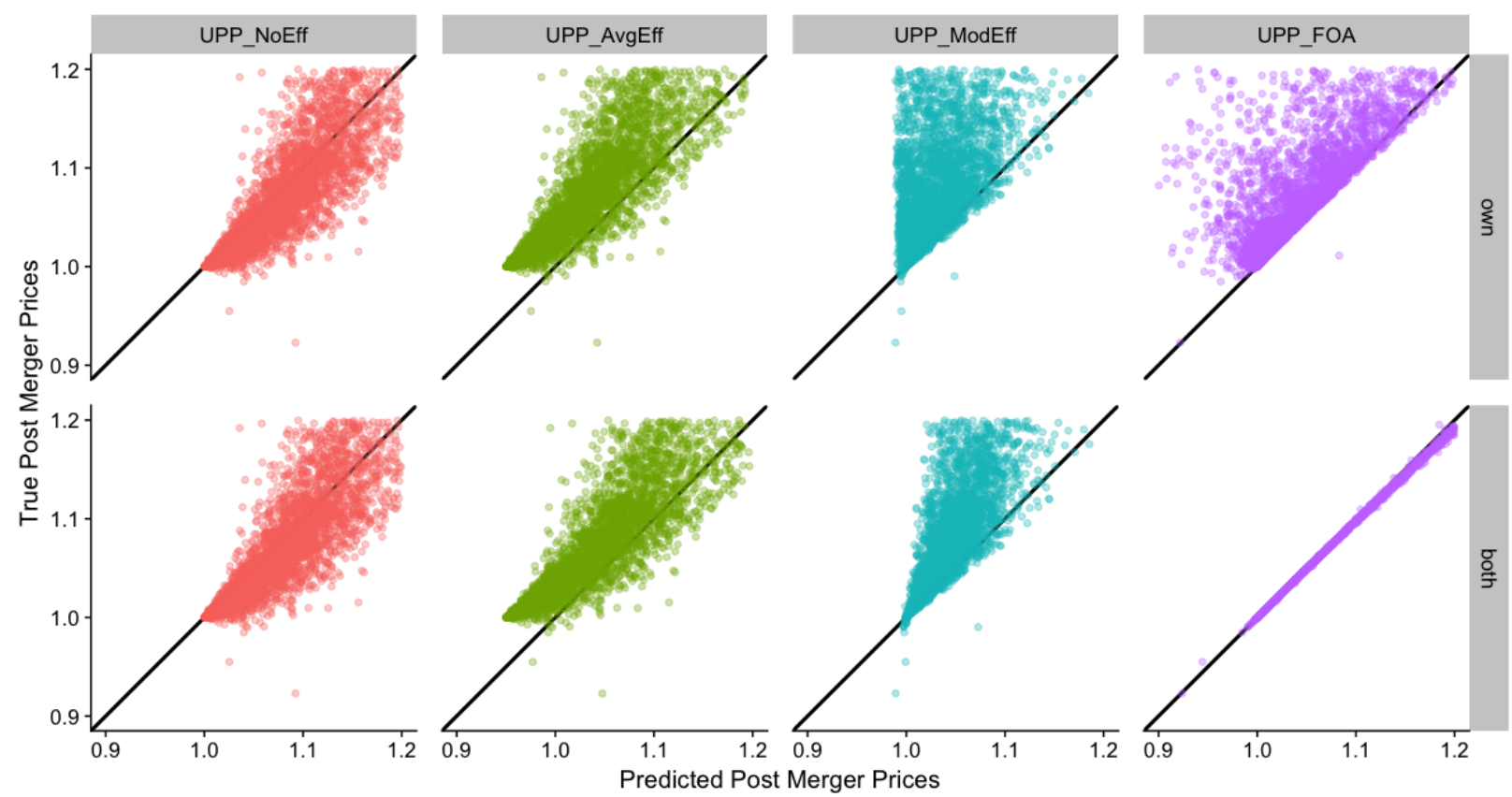

Figure 23: Accuracy of Prediction - Almost Ideal Demand, Quadratic Cost

First row shows the distribution of the true post merger prices against the predicted post merger prices using different UPP calculations and own goods' efficiencies included in the computation. Second row shows the same for both goods' efficiencies. 


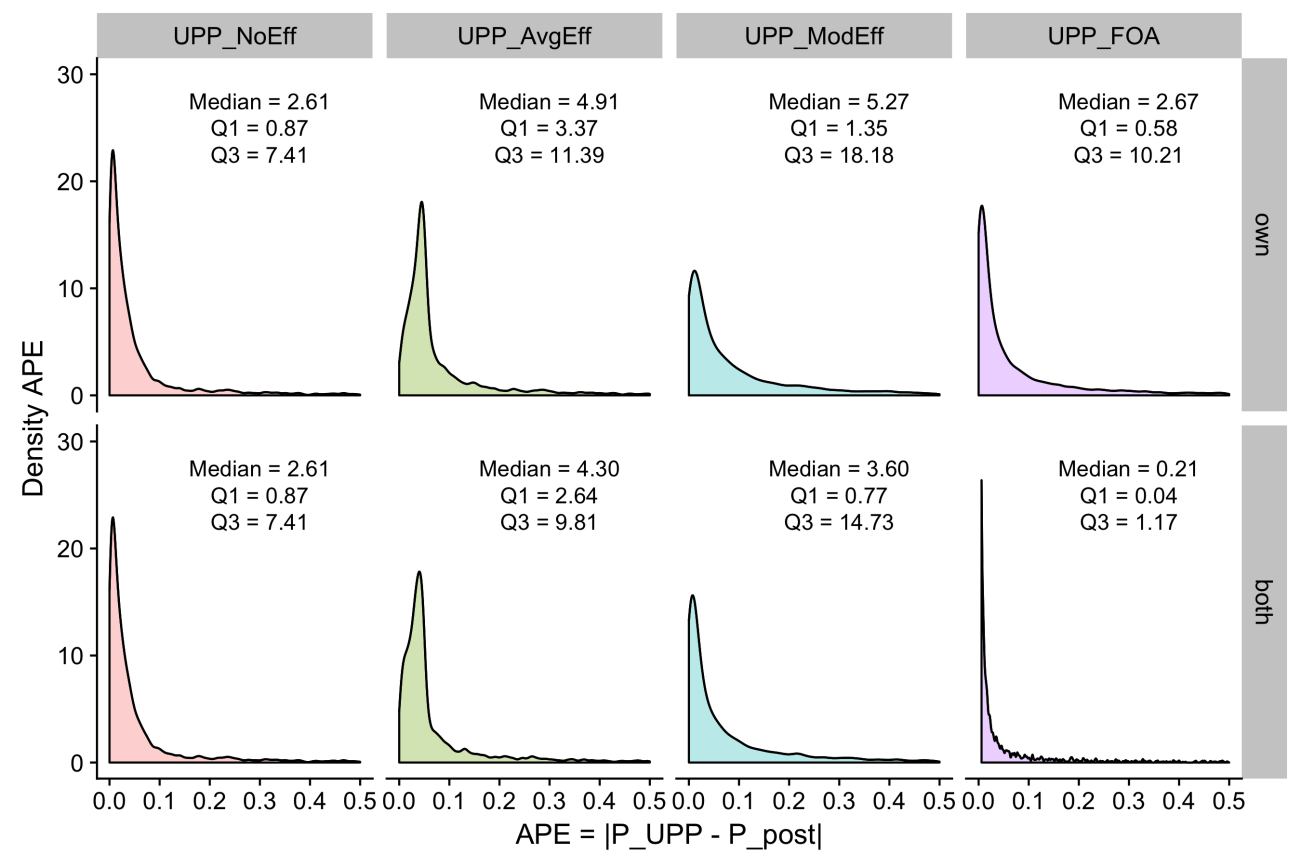

Figure 24: Absolute Prediction Errors - Almost Ideal Demand, Quadratic Cost

Portrays density kernels for absolute prediction errors, as well as the median absolute prediction error, first and third quartile for each specification..

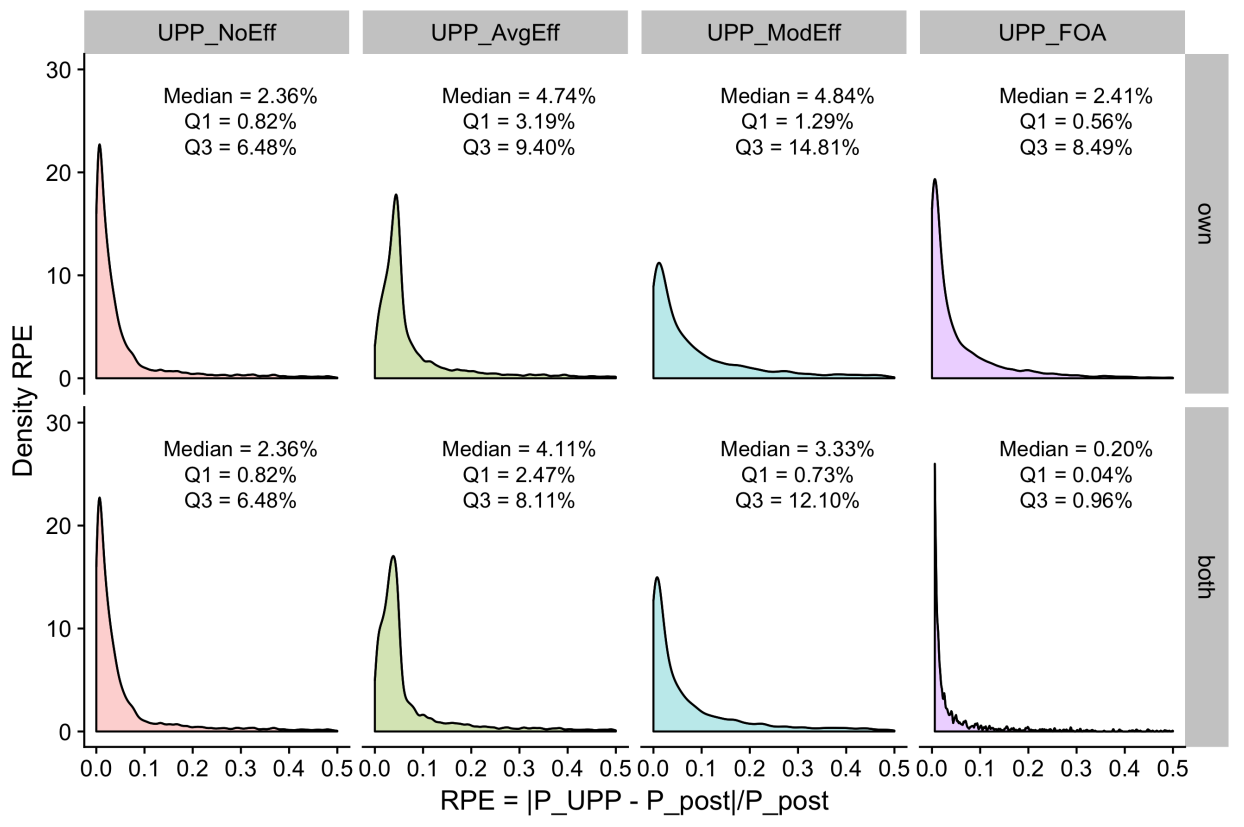

Figure 25: Relative Prediction Errors - Almost Demand, Quadratic Cost

Portrays density kernels for relative prediction errors, as well as the median relative prediction error, first and third quartile for each specification. 
Table 5: Baseline UPP with Higher Thresholds

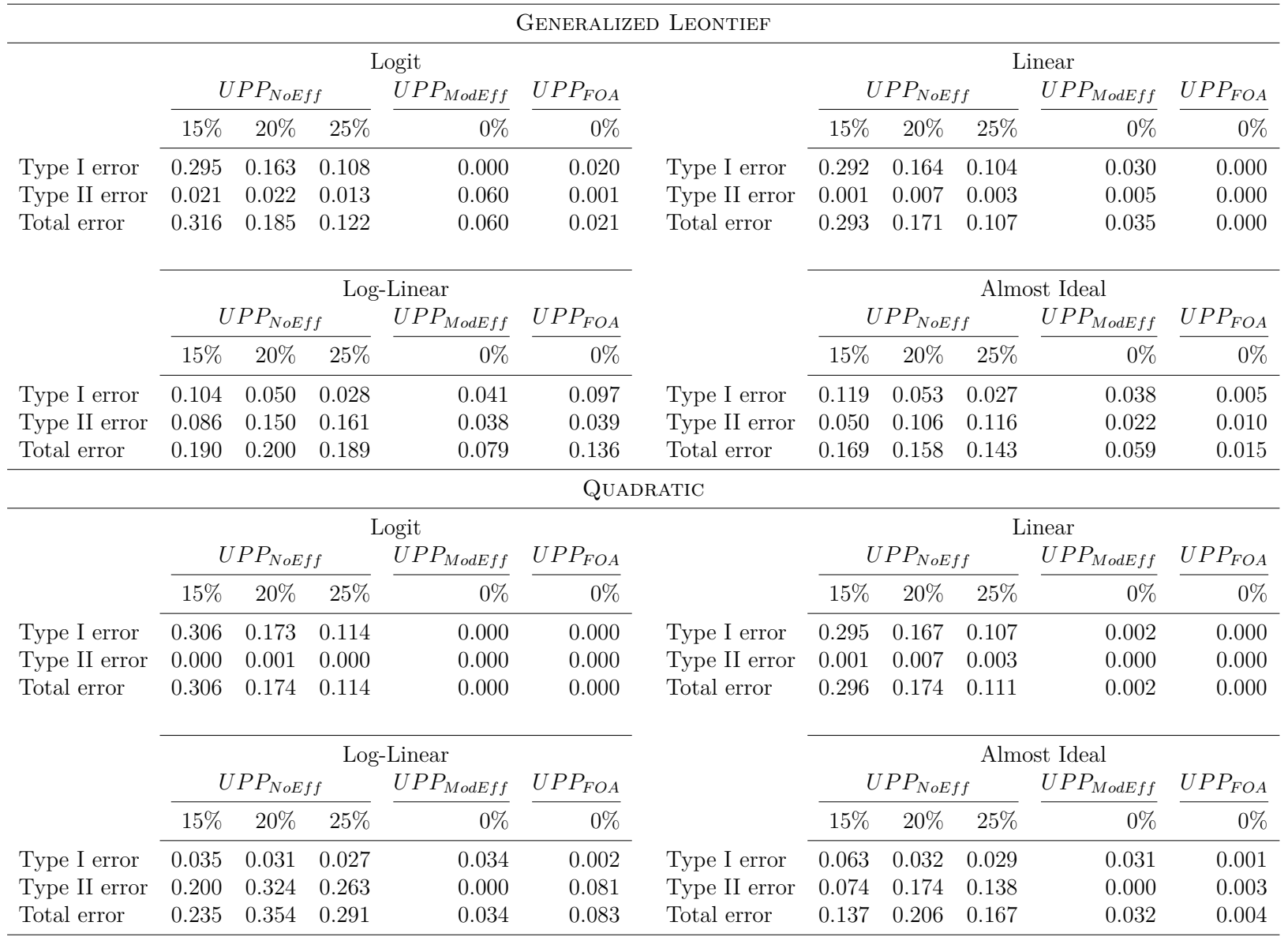

Table shows the percentage of error types in merger screening for UPP baseline formulation (for 15, 20, and $25 \%$ tolerance threshold) when compared to UPP with model-based efficiencies and first-order approximation (with a strict tolerance of $0 \%$ threshold). 
Table 6: Improvement in Merger Screening Accuracy - 10\% Tolerance Threshold

\begin{tabular}{|c|c|c|c|c|c|c|c|c|c|}
\hline \multicolumn{10}{|c|}{ Generalized Leontief } \\
\hline Logit Demand & NoEff & AvgEff & ModEff & FOA & Linear Demand & NoEff & AvgEff & ModEff & FOA \\
\hline Type I error & 0.436 & 0.123 & 0.026 & 0.007 & Type I error & 0.433 & 0.120 & 0.026 & 0.000 \\
\hline Type II error & 0.026 & 0.051 & 0.025 & 0.008 & Type II error & 0.001 & 0.026 & 0.003 & 0.000 \\
\hline Total & 0.462 & 0.175 & 0.050 & 0.015 & Total & 0.434 & 0.147 & 0.029 & 0.000 \\
\hline \multirow{2}{*}{\multicolumn{3}{|c|}{$\begin{array}{l}\text { Absolute Gain w.r.t. AvgEff } \\
\text { Relative Gain w.r.t. AvgEff (\%) }\end{array}$}} & 0.124 & 0.159 & \multirow{2}{*}{\multicolumn{2}{|c|}{$\begin{array}{l}\text { Absolute Gain w.r.t. AvgEff } \\
\text { Relative Gain w.r.t. AvgEff (\%) }\end{array}$}} & & 0.118 & 0.147 \\
\hline & & & 71.11 & 91.22 & & & & 80.56 & 100.00 \\
\hline Log-Linear Demand & NoEff & AvgEff & ModEff & FOA & Almost Ideal Demand & NoEff & AvgEff & ModEff & FOA \\
\hline Type I error & 0.224 & 0.045 & 0.008 & 0.072 & Type I error & 0.244 & 0.045 & 0.008 & 0.005 \\
\hline Type II error & 0.044 & 0.182 & 0.193 & 0.038 & Type II error & 0.024 & 0.145 & 0.138 & 0.010 \\
\hline Total & 0.267 & 0.226 & 0.201 & 0.110 & Total & 0.268 & 0.191 & 0.146 & 0.015 \\
\hline \multirow{2}{*}{\multicolumn{3}{|c|}{$\begin{array}{l}\text { Absolute Gain w.r.t. AvgEff } \\
\text { Relative Gain w.r.t. AvgEff (\%) }\end{array}$}} & 0.025 & 0.116 & \multirow{2}{*}{\multicolumn{2}{|c|}{$\begin{array}{l}\text { Absolute Gain w.r.t. AvgEff } \\
\text { Relative Gain w.r.t. AvgEff (\%) }\end{array}$}} & & 0.045 & 0.176 \\
\hline & & & 11.08 & 51.33 & & & & 23.49 & 92.24 \\
\hline \multicolumn{10}{|c|}{ QUADRATIC } \\
\hline Logit Demand & NoEff & AvgEff & ModEff & FOA & Linear Demand & NoEff & AvgEff & ModEff & FOA \\
\hline Type I error & 0.406 & 0.269 & 0.060 & 0.006 & Type & 0.412 & 0.277 & 0.068 & 0.000 \\
\hline Type II error & 0.000 & 0.002 & 0.000 & 0.000 & Type II error & 0.003 & 0.005 & 0.004 & 0.000 \\
\hline Total & 0.406 & 0.271 & 0.060 & 0.007 & Total & 0.415 & 0.283 & 0.071 & 0.000 \\
\hline \multirow{2}{*}{\multicolumn{2}{|c|}{$\begin{array}{l}\text { Absolute Gain w.r.t. AvgEff } \\
\text { Relative Gain w.r.t. AvgEff (\%) }\end{array}$}} & & 0.211 & 0.264 & \multirow{2}{*}{\multicolumn{2}{|c|}{$\begin{array}{l}\text { Absolute Gain w.r.t. AvgEff } \\
\text { Relative Gain w.r.t. AvgEff (\%) }\end{array}$}} & & 0.211 & 0.283 \\
\hline & & & 78.02 & 97.57 & & & & 74.76 & 100.00 \\
\hline Log-Linear Demand & NoEff & AvgEff & ModEff & FOA & Almost Ideal Demand & NoEff & AvgEff & ModEff & FOA \\
\hline Type I error & 0.048 & 0.033 & 0.021 & 0.031 & Type I error & 0.096 & 0.042 & 0.019 & 0.009 \\
\hline Type II error & 0.130 & 0.253 & 0.436 & 0.097 & Type II error & 0.037 & 0.122 & 0.292 & 0.004 \\
\hline Total & 0.178 & 0.286 & 0.457 & 0.128 & Total & 0.133 & 0.165 & 0.311 & 0.013 \\
\hline \multirow{2}{*}{\multicolumn{3}{|c|}{$\begin{array}{l}\text { Absolute Gain w.r.t. AvgEff } \\
\text { Relative Gain w.r.t. AvgEff (\%) }\end{array}$}} & -0.171 & 0.157 & \multirow{2}{*}{\multicolumn{2}{|c|}{$\begin{array}{l}\text { Absolute Gain w.r.t. AvgEff } \\
\text { Relative Gain w.r.t. AvgEff (\%) }\end{array}$}} & & -0.146 & 0.152 \\
\hline & & & -59.84 & 55.11 & & & & -88.60 & 92.31 \\
\hline
\end{tabular}




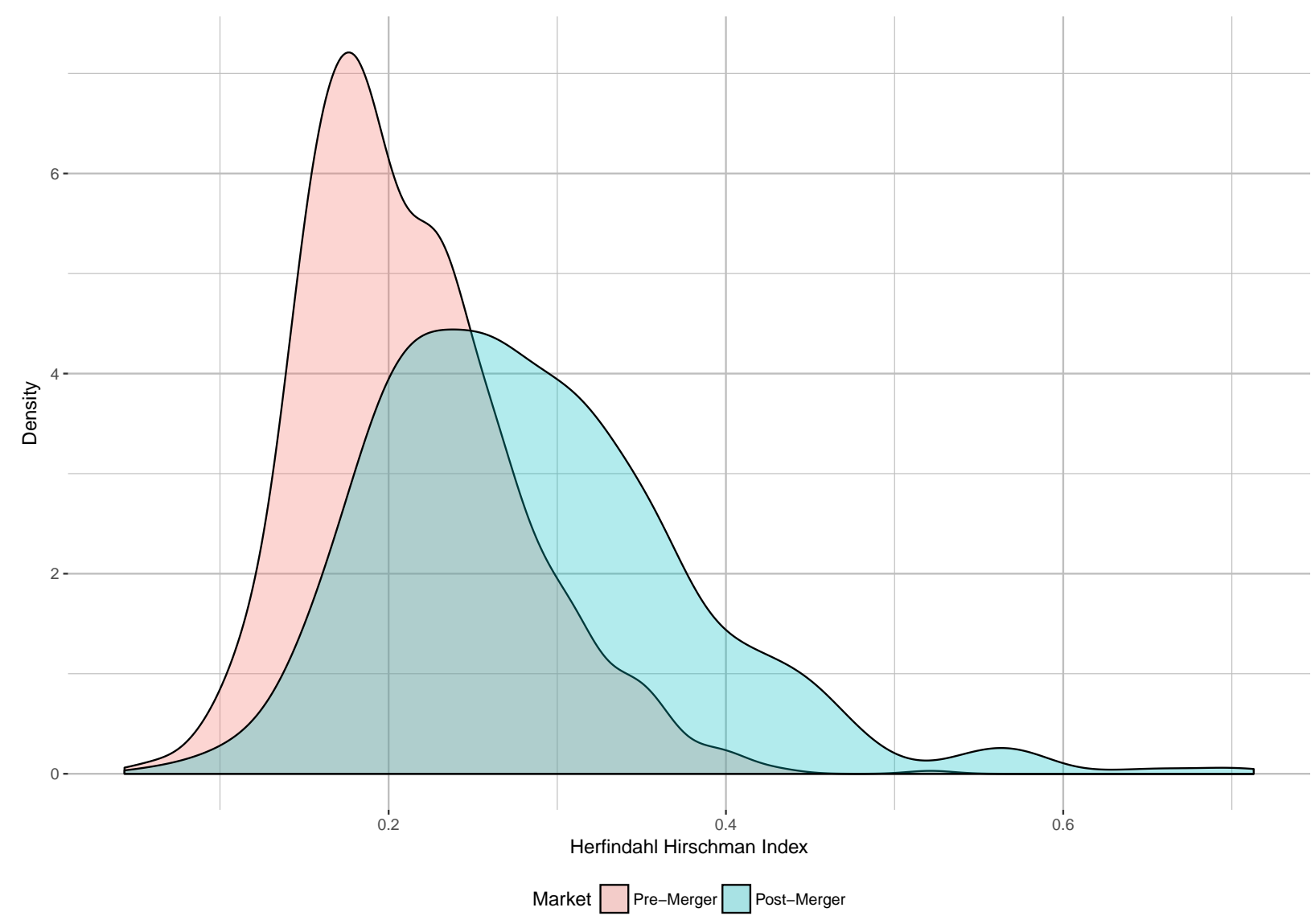

Figure 26: Market Concentration Pre and Post-Merger

Shows the distribution of market concentration within the markets pre and post merger as measured by the Herfindahl Hirschman Index. Simplifying assumption for post-merger increase in HHI is twice the product of the merging parties pre-merger market share, as used in merger screening. 